\title{
Functional Glycomics and Anxiety-related Behaviors in Single Versus Group-Housed C57BL/6 and DBA/2 Male Mice.
}

\author{
Ahmed F. Abou-Elnaga ${ }^{1}$, Gaber E. Batiha ${ }^{2}$, Christian T. Potter ${ }^{3}$, Ibrahim F. Rehan ${ }^{4 *}$
}

${ }^{1}$ Department of Husbandry and Development of Animal Wealth, Faculty of Veterinary Medicine, Mansoura University, Egypt; ${ }^{2}$ Department of Pharmacology and Therapeutics, Faculty of Veterinary Medicine, Damanhour University, Damanhour, Al Beheira, Egypt; ${ }^{3}$ Department of neuroscience, center for neuroscience at the University of Pittsburgh, Pennsylvania, United States of America; ${ }^{4}$ Department of Husbandry and Development of Animal Wealth, Faculty of Veterinary Medicine, Menofia University, Shebin Alkom, Menofia, Egypt.

\begin{abstract}
Anxiety-related behaviors are indices reflecting the welfare conditions of animals. Correlating anxiety levels of $\mathrm{C} 57 \mathrm{BL} / 6$ and $\mathrm{DBA} / 2$ in different tests to glycomic responses as a realistic biochemical markers in mice remains unclear. Sixty-four mice, 12 weeks old, were housed either singly or in groups of four ( $n=32$ each) and tested for anxiety-related behavior after 7 weeks of different housing and their anxiety levels were correlated to their functional glycomic responses in the context of different housing conditions. DBA/2 mice demonstrated less grooming, lower incidence of rearing, higher defecation, and a higher latency to begin rearing and grooming in an induced grooming test. They also displayed more anxiety-related behaviors in DL exploration than the C57BL/6 mice did. The serum $\mathrm{N}$-glycans were examined using glycoblotting along with matrix-assisted laser desportionionization-time of flight/ mass spectrometry (MALDI-TOF/MS) to follow the molecular correlates of anxiety in mice behaviors in-vivo. Sialylated $N$-glycan was significantly increased in the $\mathrm{DBA} / 2$, and correlated with the mice's behavioral data, confirming that there were more anxiety-like responses than the C57BL/6 mice. To our knowledge, sialylated $N$-glycan is a novel biomarker of anxiety. This approach has the potential to improve neurobehavioural research on anxiety.
\end{abstract}

Keywords | Anxiety, $N$-glycan, MALDI-TOF/MS, Welfare, Mice

Received | March 27, 2021; Accepted | May 29, 2021; Published | August 15, 2021

*Correspondence | Ibrahim F. Rehan, Department of Husbandry and Development of Animal Wealth, Faculty of Veterinary Medicine, Menofia University, Shebin Alkom, Menofia, Egypt; Email: ibrahim.rehan@vet.menofia.edu.eg

Citation | Elnaga AFA, Batiha GE, Potter CT, Rehan IF (2021). Functional glycomics and anxiety-related behaviors in single versus group-housed C57BL/6 and DBA/2 male mice. Adv. Anim. Vet. Sci. 9(10): 1532-1546.

DOI | http://dx.doi.org/10.17582/journal.aavs/2021/9.10.1532.1546

ISSN (Online) | 2307-8316; ISSN (Print) | 2309-3331

Copyright $\odot 2021$ Elnaga et al. This is an open access article distributed under the Creative Commons Attribution License, which permits unrestricted use, distribution, and reproduction in any medium, provided the original work is properly cited.

\section{INTRODUCTION}

$\mathrm{N}$ umerous behavioral differences have been reported between C57BL/6 and DBA/2 for memory, anxiety, pain responses, and sensitivity thresholds to psychological medications (Filiou et al., 2021). Grooming is an indicator of different internal and external states of the animal and can reflect anxiety (Kalueff et al., 2016; Jirkof et al., 2019).

In the induced grooming test, latency, frequency of bouts, duration, rearing behavior, and defecation are the main indices to assess anxiety-related behavior (Kalueff et al., 2016). Dark light (DL) exploration is another common etiological model of anxiety-related behavior that is designed for mice (Eltokhi et al., 2020).

In our study, we used both tests to confirm the level of anxiety between both $\mathrm{C} 57 \mathrm{BL} / 6$ and $\mathrm{DBA} / 2$ strains in the different housing conditions.

Moreover, we analyzed the mouse's sera, which contained both Neu5Ac and Neu5Gc-sial-terminal(s) due to the 
effect of CMP-Neu5AcHydroxylase enzyme, that converts Neu5Ac to Neu5Gc (Mahmoud et al., 2019; Rehan et al., 2020). These sialylated Neu5Ac/Neu5Gc-terminal(s) $\mathrm{N}$-glycans were differently expressed in stress conditions (Mahmoud et al., 2019; Rehan et al., 2020). However, Neu5Gc-terminal(s) also maintains the anxiety-adapted responses in BALB/c mice strain (Gebrehiwot et al., 2018; Mahmoud et al., 2019; Rehan et al., 2020). Additionally, lectin-binding sia- $\alpha-2-6 \mathrm{Gal} / \mathrm{GalNAc}$ was elevated in mice under chronic stress and in depressed patients, whereas ST6GalNAc2 expression was diminished in blood sampling taken from depressed patients (Yamagata et al., 2018).

Thus, we hypothesize that the expression levels of these two sialic acid terminal $N$-glycan determined using glycoblotting combined with matrix-assisted laser desportion ionization-time of flight/mass spectrometry (MALDI-TOF/ MS) (Miura et al., 2008; Hirose et al., 2011; Sanes et al., 2018; Mahmoud et al., 2019; Rehan et al., 2020) can be used as a key to estimating anxiety in-vivo. In the present study, we investigate the effect of single housing on anxiety-related behavior (induced grooming test, DL exploration test) correlating to their functional glycomic profiles in two different strains.

\section{MATERIALS AND METHODS}

All experimental procedures were conducted following the Menofia University legislation and guidelines. In addition, all animal experiments reported here were approved by the Ethical Committee of Menofia University, Egypt (Protocol dated 032021).

\section{General Animal Housing}

Sixty-four male mice, (C57BL/6J and DBA/2, $n=32$ each), 12 weeks old, $28 \pm 2.1$ grams upon arrival were used in this experiment. They were obtained from Theodor Bilharz Research Institute, Giza, Egypt. All mice were maintained under controlled temperature conditions $\left(22 \pm 2{ }^{\circ} \mathrm{C}\right)$, humidity (60\%), lighting regime (a 12:12 h light/dark cycle), and had access to water and pelleted food ad-libitum (mice chow ${ }^{\varpi}$, Oil and Soap Manufacturing Company, E1-Gharbia, Egypt). The bedding was changed weekly, and nesting material (wood shavings) was used as enrichment for all mice.

\section{EXPERIMENTAL TREATMENTS}

At arrival, according to (Võikar et al., 2005) mice from the two strains were assigned randomly to one of the following experimental treatments for 7 consecutive weeks:

1. "Group housing" $(\mathrm{GH}): 16$ mice of C57BL/6J strain and 16 mice of DBA/2 strain were housed in groups of four in Plexiglas cages $(27.5 \mathrm{~cm}$ length $\times 16.5 \mathrm{~cm}$ width $\times 21.5 \mathrm{~cm}$ height).

2. "Single housing" (SH): 16 mice of C57BL/6J strain and 16 mice of DBA/2 strain were housed singly in the same size as the Plexiglas cages

\section{Spray Test (Induced-Grooming Test)}

For induction of artificial grooming, mice were transported to the dimly lit room and left undisturbed for $3 \mathrm{hrs}$ before the testing, the mice were individually placed in a clean plastic observation box $(30 \mathrm{~cm} \times 30 \mathrm{~cm} \times 30 \mathrm{~cm})$, misted with a spray bottle filled with water $\left(25^{\circ} \mathrm{C}\right)$, were placed in front of the spray nozzle $20-30 \mathrm{~cm}$ away, and lightly sprayed from above eight times to the dorsal surface of mice by the mist. The box of observations was thoroughly cleaned with ethanol 70\% after each subject finishes the test. In all experiments, each mouse has a 5 minutes recording with a digital video camera (Hikvision, Binjiang District, Hangzhou, China). During the recording time, the experimenter stands $2 \mathrm{~m}$ away and in front of the observation box.

To evaluate grooming activity, three behavioral measures were assessed in every test as follows: 1) latency to start grooming (s), 2) frequency (bout), and 3) duration spent grooming (s). Also, three non-grooming measures had been evaluated; 1) vertical rears (total duration for animal stand vertical on its hind legs and his forelegs are in the air or against box wall),2) the latency to initiate vertical rear (s), and 3) defecation. The number of deposited boli was scored as an index of their emotion (Kalueff et al., 2004).

\section{Dark Light (DL) Exploration Test}

Mice were tested in a Plexiglass box $(45 \mathrm{~cm}$ length $\mathrm{x} 20 \mathrm{~cm}$ width $\mathrm{x} 20 \mathrm{~cm}$ height) that had two compartments. Twothirds of the box was illuminated by 500 Lux, while the other $1 / 3$ was entirely darkened by black paint. Both compartments were separated by a door $(10 \mathrm{~cm} \times 5 \mathrm{~cm})$. At the beginning of the test, animals were placed on the dark side for 1 minute. After this first minute, the door was opened and the mice had the chance to walk free in between both compartments for 5 minutes. Latency to enter the illuminated side, time spent in both compartments, and frequency to enter the light compartment were calculated by an experienced experimenter (Eltokhi et al., 2020).

The order of the tests (and intervals between them) was: induced grooming, then 2 days of rest, then DL. The order of testing was chosen to conduct the less invasive tests first (Võikar et al., 2004). All behavioral tests were performed during the dark period of the circadian cycle.

Importantly, the serum $\mathrm{N}$-glycan levels of mice were analyzed immediately after performing the behavioral tests to confirm the in vivo status of the experimented mice. 


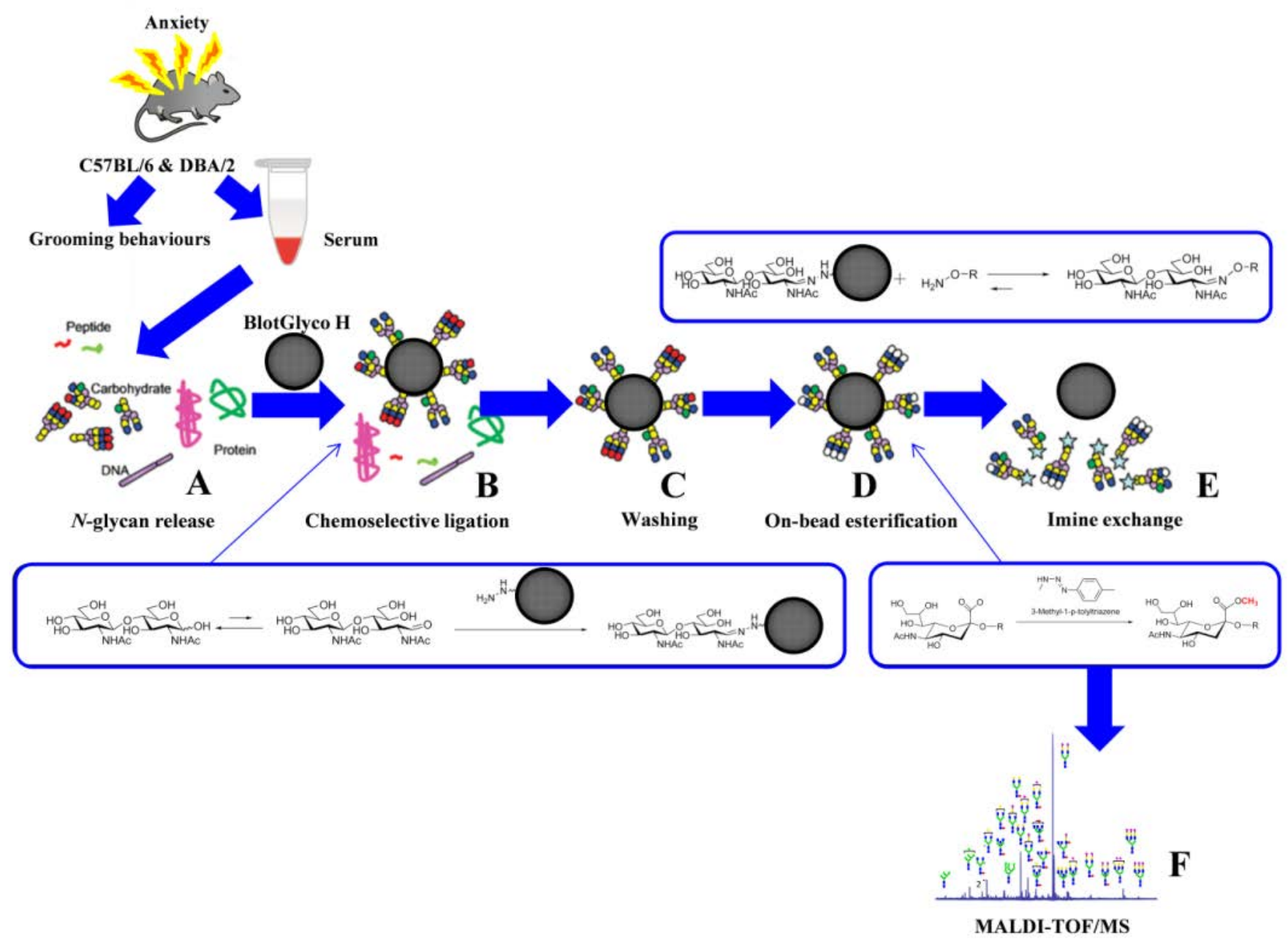

Figure 1: Glycoblotting followed by MALDI-TOF/MS analysis of mice sera: (A) $N$-glycan release; (B) chemoselective ligation of whole serum $N$-glycans by "BlotGlyco" beads; (C) washing; (D) on-bead esterifications of sialyl-terminal(s); and (E) trans-iminization by benzyloxiamine to generate BOA-tagged $N$-glycans; and (F) MALDI-TOF/MS analysis. The figure was drawn, in its original form, by the authors of this manuscript.

\section{Glycoblotting-Based Serum Glycomics}

The experimental mice were decapitated for euthanasia, and $0.5-1.0 \mathrm{ml}$ of the blood was carefully collected. Further, we kept the clotted blood at $4{ }^{\circ} \mathrm{C}$ for $16 \mathrm{hr}$ and proper centrifugation was performed at $5000 \mathrm{~g}$ for up to10 minutes. Finally, mice sera were transferred into new tubes to keep them at $-80^{\circ} \mathrm{C}$ until they were analyzed. Ten microliters of mice sera ( $n=32 /$ group) were prepared for the release of $N$-glycans. The $N$-glycan mixture underwent the glycoblotting process for the quantification before the MALDI-TOF/MS analysis. The human $N$-glycan sera protocol, with simple modifications, was used in this study, as reported previously (Gizaw et al., 2015; Gizaw et al., 2016; Gebrehiwot et al., 2018; Mahmoud et al., 2019; Rehan et al., 2020). Afterward, a PNGase F. BlotGlyco ${ }^{\circ} \mathrm{H}$ bead was used to capture $\mathrm{N}$-glycan carefully by the glycoblotting method. The methyl-esterification of the sialic acid terminal(s) was also performed. Then, to tag $N$-glycan with benzyloxyamine (BOA), a trans-imination reaction was done. The mixture of $N$-glycans tagged with $\mathrm{BOA}$ was subjected to MAL-
DI-TOF/MS analysis (as shown in Figure $1 \mathrm{~A}-\mathrm{F}$ ). Importantly, the GlycoMod Tool predicted the possible $N$-glycan compositions of the experimental masses, which were mainly reported on GlycoSuiteDB, (http://web.expassy. $\mathrm{org} / \mathrm{glycomod} /$ ), and non-reported compositions were later checked using the consortium for functional glycomics (CFG), (http://www.functionalglycomics.org), to identify and categorize the genetic diversity in $N$-glycan structures in mouse serum. $N$-glycan profiling was primarily done on sources from the sera of Ratus ratus, especially sources from mouse serum, cells, and tissues. Moreover, the intensity of the isotopic peak of each glycan was normalized using 40 $\mu \mathrm{M}$ of the internal standard (disialyloctasaccharide) for each status, and their concentrations were calculated from a calibration curve using human serum standards) Gizaw et al., 2015; Gizaw et al., 2016; Gebrehiwot et al., 2018; Mahmoud et al., 2019; Rehan et al., 2020). The observed ions of experimental $\mathrm{m} / \mathrm{z}$ values which were performed using MALDI-TOF/MS were relatively higher than the Expasy MW values because $N$-glycans were labeled 


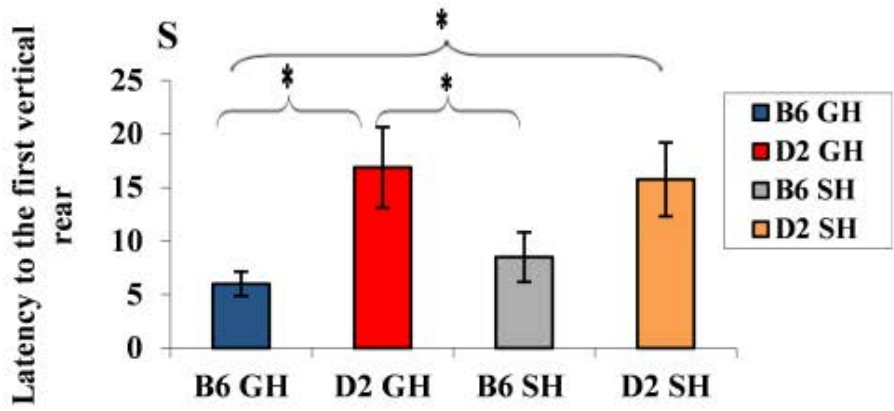

Treatment

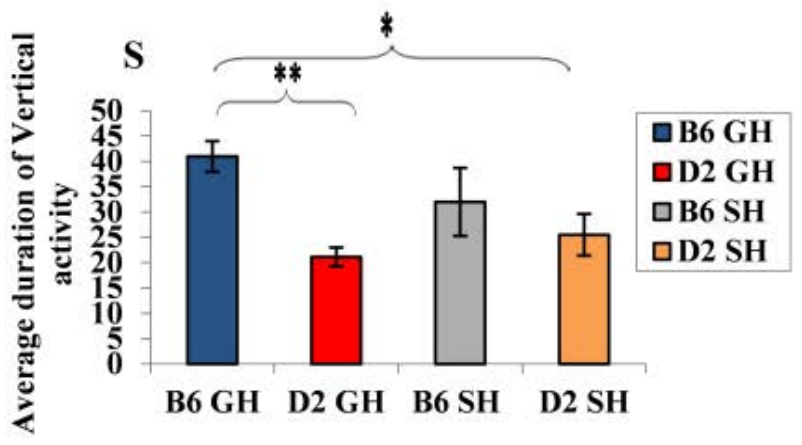

Treatment

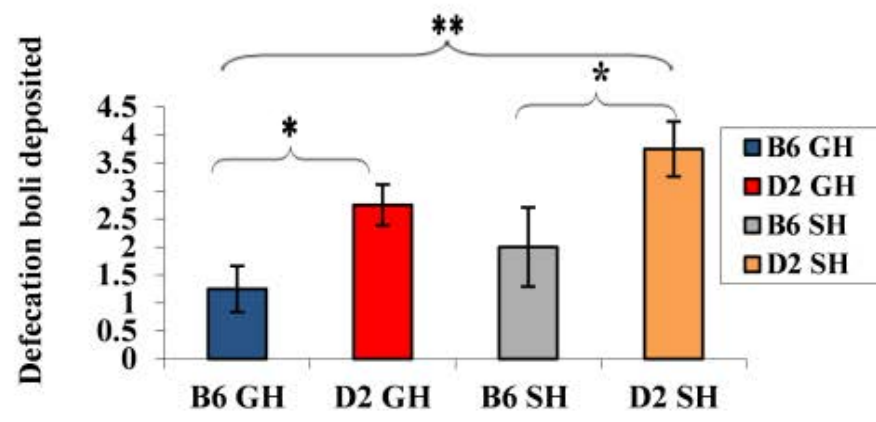

Treatment

Figure 2: Average latency of first vertical rear, average duration of vertical activity, and number of fecal boli deposited by the mice of the two inbred stain of experimental treatments. Data represented as mean \pm SEM."****" showed a significance level, $P \leq 0.001$, ,***" a significance level, $P \leq 0.01$, ”* a significance level, $P \leq 0.05$ ). B6GH: GH-C57BL/6J; D2GH: GH-DBA/2; B6SH: SH-C57BL/6J; D2SH: SH-DBA/2; S: second.

with BOA for trans-imination $(105 \mathrm{~m} / \mathrm{z})$, formation of $\mathrm{Na}$-adduct $(23 \mathrm{~m} / \mathrm{z})$ by laser condition during shooting for performing Two-way ANOVA of SPSS version 20 was performed using strain (C57BL/6J and DBA/2) and housing (group- and single-housed) as the independent variables for detecting the respective effects. Post hoc comparisons were carried out using the Bonferroni test. The $\mathrm{N}$-glycan concentration $(\mu \mathrm{M})$ for each molecular mass $(\mathrm{m} / \mathrm{z})$ was fixed as the dependent variable followed by Bonferro$n i$-Post Hoc tests to compare each strain to its housing type. The significant difference was considered when $P$ $\leq 0.05$. Also, the Pearson coefficient correlation test for the duration of grooming and Spearman's rbo correlation test for grooming bouts had been applied to estimate the correlation between $\mathrm{N}$-glycans and different behaviors. In which $(r)=0.7$ was considered a strong correlation, $(r)=0.5-0.7$ a moderate to strong correlation, and $(r)=$ 0.3-0.5 a weak to moderate correlation.

MS analysis, and the esterification of sialic acid(s) per methyl group $(14 \mathrm{~m} / \mathrm{z})$. More detailed information of chemicals and $\mathrm{N}$-glycomic steps, written in Supporting Information I, II.

\section{Statistical Analyses}

Two-way ANOVA of SPSS version 20 was performed using strain $(\mathrm{C} 57 \mathrm{BL} / 6 \mathrm{~J}$ and $\mathrm{DBA} / 2)$ and housing (groupand single-housed) as the independent variables for detecting the respective effects. Post hoc comparisons were carried out using the Bonferroni test. The N-glycan concentration $(\mu \mathrm{M})$ for each molecular mass $(\mathrm{m} / \mathrm{z})$ was fixed as the dependent variable followed by Bonferroni-Post Hoc tests to compare each strain to its housing type. The significant difference was considered when $P \leq 0.05$. Also, the Pearson coefficient correlation test for the duration of grooming and Spearman's rho correlation test for grooming bouts had been applied to estimate the correlation between $N$-glycans and different behaviors. In which $(r)=0.7$ was considered a strong correlation, $(r)=0.5-0.7$ a moderate to strong correlation, and $(r)=0.3-0.5$ a weak to moderate correlation.

\section{RESULTS AND DISCUSSION}

\section{BeHAVIORAL Findings}

The data demonstrated the strain, housing condition, and strain by housing condition interaction effect to clarify all factors that might cause such changes in mice's behaviors. We predicted that such behavior would not be changed ei 

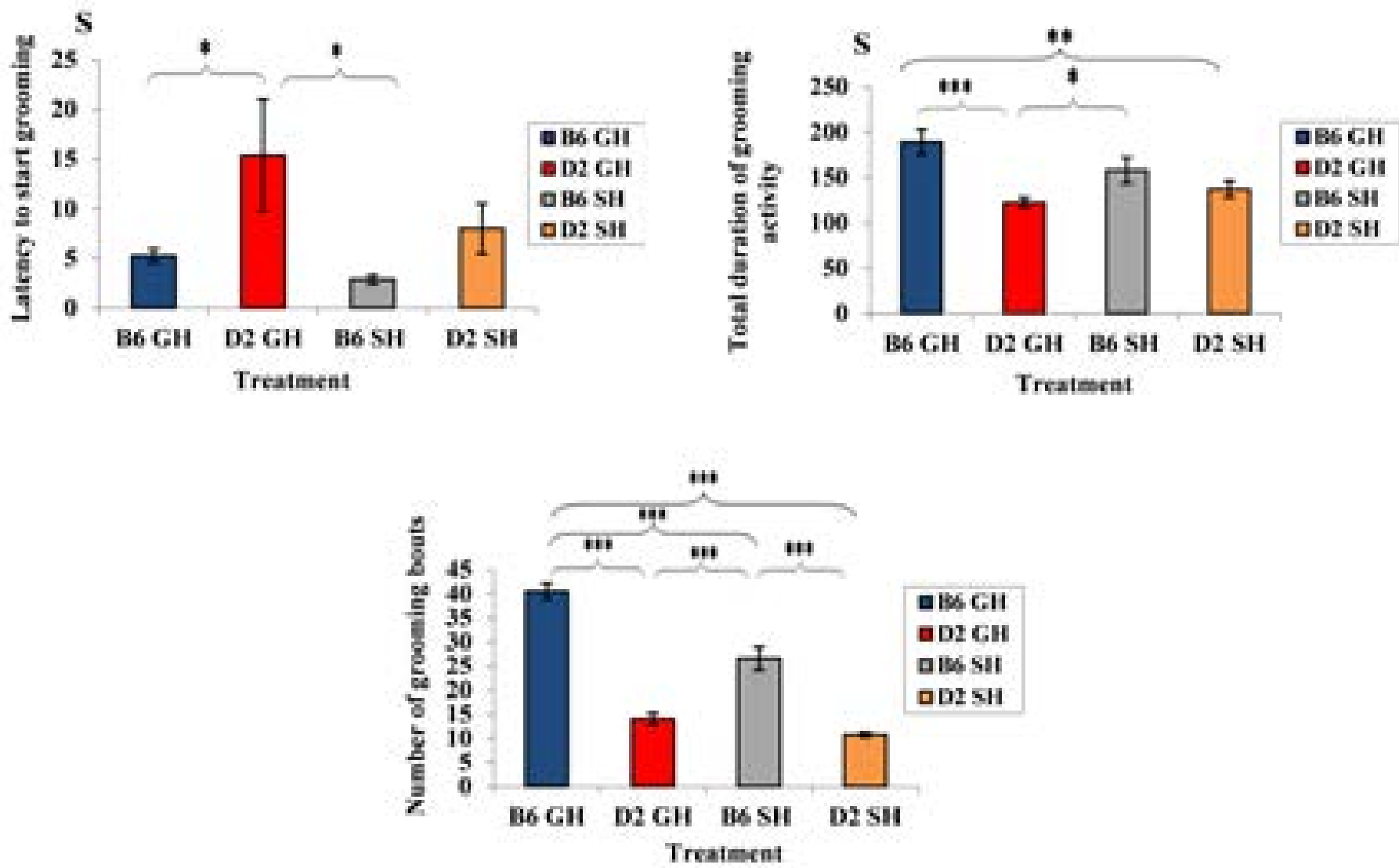

Figure 3: Average latency to start grooming, average of total duration of grooming activity, and average number of grooming bouts by the mice of the two inbred strains of experimental treatments. Data represented as mean \pm SEM.”***" showed a significance level, $P \leq 0.001$,"**" a significance level, $P \leq 0.01$,"*” a significance level, $P \leq 0.05$ using SPSS program). B6GH: GH-C57BL/6J; D2GH: GH-DBA/2; B6SH: SH-C57BL/6J; D2SH: SH-DBA/2; S: second.

ther due to the genetic background or the special housing condition only, but due to handling a specific strain in a particular housing condition.

Strain effect: as shown in Figure 2, GH-C57BL/6J mice demonstrated shorter latency to start vertical rearing than those of GH-DBA/2 mice (latency: 6s (C57BL/6J), 16.8s $\left.(\mathrm{DBA} / 2), F_{(3,60)}=10.0508, P=0.003\right)$. Moreover, the average number of rears increased in $\mathrm{GH}-\mathrm{C} 57 \mathrm{BL} / 6 \mathrm{~J}$ than in $\mathrm{GH}-$ $\mathrm{DBA} / 2$ (average number of rears: $41 \mathrm{r}(\mathrm{GH}-\mathrm{C} 57 \mathrm{BL} / 6 \mathrm{~J})$, $\left.21.1 \mathrm{r}(\mathrm{GH}-\mathrm{DBA} / 2), F_{(3,60)}=9.335, P=0.004\right)$. A strain effect was also noticed in deposited boli by mice. For instance, $\mathrm{DBA} / 2$ mice, in both housing conditions, defecated more than C57BL/6J ones (defecation average: $1.25 \mathrm{~d}(\mathrm{GH}-$ C57BL/6J), $2.75 \mathrm{~d}(\mathrm{GH}-\mathrm{DBA} / 2), 2 \mathrm{~d}(\mathrm{SH}-\mathrm{C} 57 \mathrm{BL} / 6 \mathrm{~J})$, $\left.3.75 \mathrm{~d}(\mathrm{SH}-\mathrm{DBA} / 2), F_{(3,60)}=10.11, P=0.003\right)$. In Figure 3, induced grooming behavior patterns showed a significant strain effect where GH-DBA/2 mice displayed more latency to start grooming compared to GH-C57BL/6J (latency: $5.25 \mathrm{~s}(\mathrm{C} 57 \mathrm{BL} / 6 \mathrm{~J}), 15.3 \mathrm{~s}(\mathrm{DBA} / 2), F_{(3,60)}=5.9843$, $P=0.020)$. In addition, the total duration of grooming activity was greater in $\mathrm{GH}-\mathrm{C} 57 \mathrm{BL} / 6 \mathrm{~J}$ compared to $\mathrm{GH}-$ DBA $/ 2$ mice, $F_{(3,60)}=15.238, P=0.002$. For the number of grooming bouts, C57BL/6J performed more grooming bouts than those DBA/2 mice in both housing conditions, (average of grooming bouts: $40.5 \mathrm{~b}(\mathrm{GH}-\mathrm{C} 57 \mathrm{BL} / 6 \mathrm{~J}), 14$ b (GH-DBA/2), 26.5 b (GH-C57BL/6J), 10.75 b (GH$\left.\mathrm{DBA} / 2), F_{(3,60)}=176.770, P=0.0001\right)$. In Figure 4, C57BL/6J spent more time in the light compartment when compared to DBA/2 mice in both housing conditions, $F_{(3,60)}=7.419$, $P=0.022$. Also, we found that strain had a significant effect on latency to enter the light compartment, where C57BL/6J entered the light side faster than DBA/2 strain in both housing $F_{(3,60)}=9.543, P=0.0013$.

Housing condition effect: $\mathrm{GH}-\mathrm{C} 57 \mathrm{BL} / 6 \mathrm{~J}$ mice exhibited more grooming bouts than $\mathrm{SH}-\mathrm{C} 57 \mathrm{BL} / 6 \mathrm{~J}$ (grooming bouts: $40.5 \mathrm{~b}(\mathrm{GH}-\mathrm{C} 57 \mathrm{BL} / 6 \mathrm{~J}), 26.5 \mathrm{~b}(\mathrm{SH}-\mathrm{C} 57 \mathrm{BL} / 6 \mathrm{~J})$, $\left.F_{(3,60)}=29.466, P=0.0003\right)$. Furthermore, single housing increased latency to enter a light compartment in DBA/2, $F_{(3,60)}=9.543, P=0.05$ in DL box. It indicated that SHDBA/2 displayed significantly less anxiety than C57BL/6J of both housing and GH-DBA/2 mice, see Figure 4.

Strain*housing condition effect: $\mathrm{SH}-\mathrm{C} 57 \mathrm{BL} / 6 \mathrm{~J}$ exhibited more grooming bouts than $\mathrm{GH}-\mathrm{DBA} / 2$ mice, while, GH-C57BL/6J exhibited more grooming bouts than $\mathrm{SH}-$ DBA/2 (grooming bouts: $40.5 \mathrm{~b}(\mathrm{GH}-\mathrm{C} 57 \mathrm{BL} / 6 \mathrm{~J}), 26.5 \mathrm{~b}$ (SH-C57BL/6J), 14b(GH-DBA/2), 10.75b(SH-DBA/2), $\left.F_{(3,60)}=11.4438, P=0.002\right)$. In addition, we investigated 

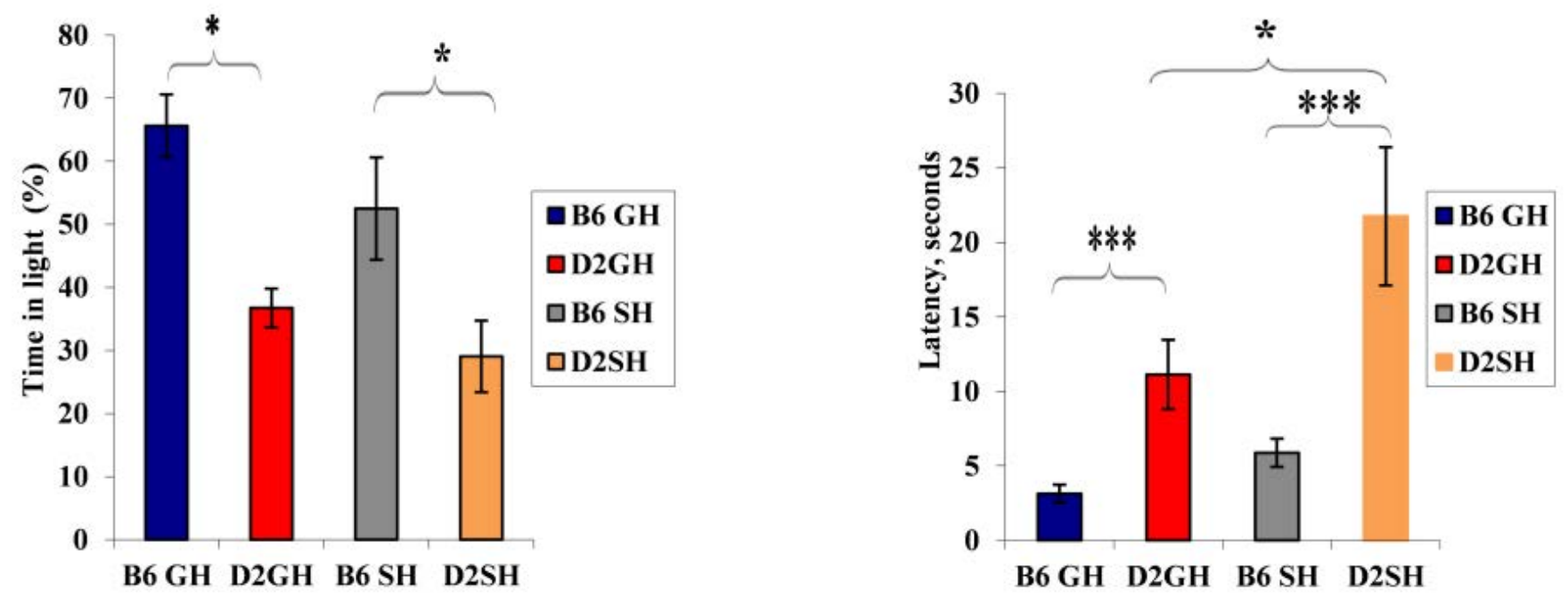

Figure 4: Time (\%) in light litcompartment, latency in seconds to enter light compartment in DL box by the mice of the two inbred strains in the two experimental treatments. Data represented as mean \pm SEM"***" showed a significance level, $P \leq 0.001$, "**” a significance level, $P \leq 0.01$,"*” a significance level, $P \leq 0.05$ using SPSS program). B6GH:GH-C57BL/6J; D2GH:GH-DBA/2; B6SH:SH-C57BL/6J; D2SH:SH-DBA/2; S: second.

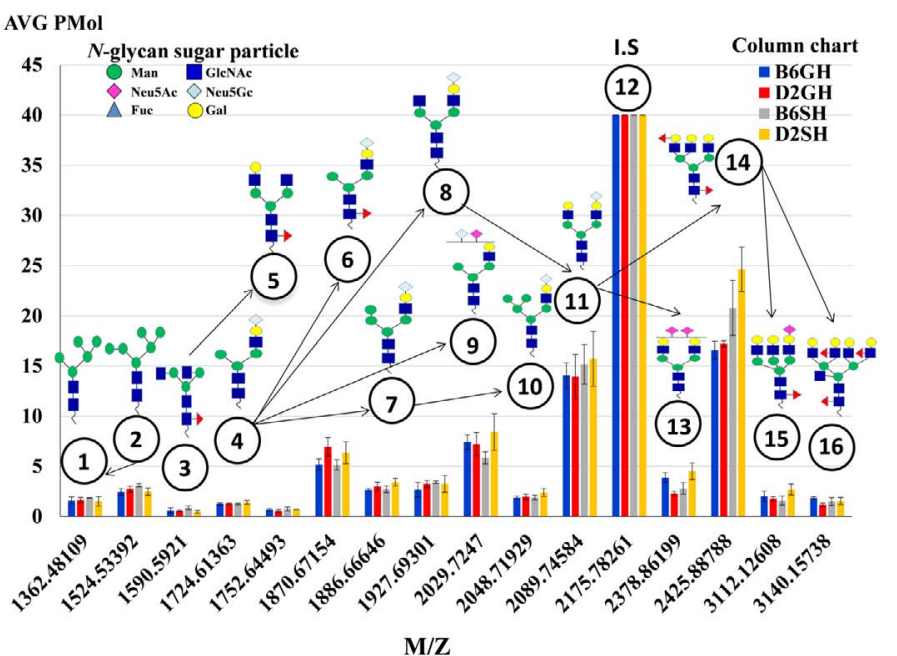

Figure 5: The average expression levels (pmole) of the 15 major $N$-glycans of the C57BL/6 and DBA/2 mouse serum, identified by the biosynthetic pathway. Peak number 12 at $\mathrm{m} / \mathrm{z} 2175$ assigned as an internal standard structured, $\left[(\mathrm{Hex})_{2}(\mathrm{HexNAc})_{2}\right.$ $\left.(\mathrm{Neu} 5 \mathrm{Ac})_{2}+(\mathrm{Man})_{3}(\mathrm{GlcNAc})_{1}\right]$, with $40 \mu \mathrm{M}$ concentrations. $\mathrm{N}$-glycans sugar particles showed as follow: HexNAc, $\mathrm{N}$-acetylhexosamine (GlcNAc, $\mathrm{N}$-acetylglucoseamine (blue square) or GalNAc, $\mathrm{N}$-acetylgalactoseamine (yellow square) depends on the description); Deoxyhexose, fucose (redtriangle); Hex, hexose (Mannose (green circle), and galactose (yellow circle) depend on the description); and Neu5Ac, 5- $\mathrm{N}$-acetylneuraminic acid (purple diamond), I.S, Internal Standard. B6GH:GHC57BL/6J; D2GH: GH-DBA/2; B6SH: SH-C57BL/6J; D2SH: SH-DBA/2.

the strain*housing condition effect and showed that GH$\mathrm{DBA} / 2$ mice had more latency to groom when compared to SH-C57BL/6J (latency: 2.8 s (GH-DBA/2), 15.3 s (SH-
C57BL/6J), $\left.F_{(3,60)}=1.643, P=0.042\right)$. We found that $\mathrm{SH}-$ $\mathrm{C} 57 \mathrm{BL} / 6 \mathrm{~J}$ mice groomed more time than $\mathrm{GH}-\mathrm{DBA} / 2$; whereas $\mathrm{GH}-\mathrm{C} 57 \mathrm{BL} / 6 \mathrm{~J}$ groomed more time than $\mathrm{SH}$ $\mathrm{DBA} / 2$, (grooming duration: 157.7 s (SH-C57BL/6J), $122.5 \mathrm{~s}(\mathrm{GH}-\mathrm{DBA} / 2), 137 \mathrm{~s}(\mathrm{SH}-\mathrm{DBA} / 2), F_{(3,60)}=4.20057$, $P=0.049$ ).

\section{Mass SPECTROMetry ANALYSES OF N-GLyCANS}

The $N$-glycomic analysis procedure in C57BL/6 and DBA $/ 2$ mice sera was performed using glycoblotting accompanied with MALDI-TOF/MS (Figure 1, steps A F). The selected $15 \mathrm{~N}$-glycans structures (Table 1 ) of mice sera were identified in Glycosuit DB and/or the Consortium for Functional Glycomics (CFG). The ratio of $N$-glycan in mouse (C57BL/6 and DBA/2) serum showed $13 \%$ high mannose-, $34 \%$ hybrid-, and $53 \%$ of complex sialylated $N$-glycans. Importantly, the two sial-terminals Neu5Ac andNeu5Gc, synthesized in both mice sera, $N$-glycomics can be assessed by the expression of $N$-glycan MS spectra peaks in both mice strains that were significantly altered in all experimental groups (Figure 5). Herein, the significances of these peaks were statistically calculated among groups (Table 2).

\section{N-glycan Peaks Analysis}

For strain effect, it was obvious that DBA/2 mice, especially single housed ones, were very responsive to the changes in $N$-glycans when compared to C57BL/6 mice. As a result, shown in Table 2, we noticed changes in most of our estimated peaks indicating that SH-DBA/2 mice showed more significant elevation in peaks $4,7,9,10,13,15$ when compared to SH-C57BL/6, $F_{(3,60)}=1.822, P=0.006$, $F_{(3,60)}=4.862, P=0.009, F_{(3,60)}=3.432, P=0.016, F_{(3,60)}=5.238$, 
Table 1: Estimated Structure of 15 Major $N$-glycans of Serum in C57BL/6 and DBA/2 Mice.

\begin{tabular}{|c|c|c|c|c|c|}
\hline $\begin{array}{l}\text { Peak } \\
\text { number }\end{array}$ & $\mathbf{m} / \mathbf{z}$ & ExPasy MW & Structure & Type & Source \\
\hline (a) 1 & 1362.48109 & 1234.43347 & $(\mathrm{Hex})_{2}+(\mathrm{Man})_{3}(\mathrm{GlcNAc})_{2}$ & High-mannose & UniCarbKB \\
\hline (a) 2 & 1524.53392 & 1396.4863 & $(\mathrm{Hex})_{3}+(\mathrm{Man})_{3}(\mathrm{GlcNAc})_{2}$ & High-mannose & UniCarbKB \\
\hline (a) 3 & 1590.5921 & 1462.54448 & $(\mathrm{HexNAc})_{2}(\text { Deoxyhexose })_{1}+(\mathrm{Man})_{3}(\mathrm{GlcNAc})_{2}$ & Complex & UniCarbKB \\
\hline (b) 4 & 1724.61363 & 1582.55036 & $(\mathrm{Hex})_{1}(\mathrm{HexNAc})_{1}(\mathrm{Neu} 5 \mathrm{Gc})_{1}+(\mathrm{Man})_{3}(\mathrm{GlcNAc})_{2}$ & Hybrid & UniCarbKB \\
\hline (a) 5 & 1752.64493 & 1624.59731 & $(\mathrm{Hex})_{1}(\mathrm{HexNAc})_{2}(\text { Deoxyhexose })_{1}+(\mathrm{Man})_{3}(\mathrm{GlcNAc})_{2}$ & Complex & UniCarbKB \\
\hline (c) 6 & 1870.67154 & 1728.60827 & $\begin{array}{l}(\mathrm{Hex})_{1}(\mathrm{HexNAc})_{1}(\text { Deoxyhexose })_{1}(\mathrm{Neu} 5 \mathrm{Gc})_{1}+(\mathrm{Man})_{3} \\
(\mathrm{GlcNAc})_{2}\end{array}$ & Hybrid & UniCarbKB \\
\hline (b) 7 & 1886.66646 & 1744.60319 & $(\mathrm{Hex})_{2}(\mathrm{HexNAc})_{1}(\mathrm{Neu} 5 \mathrm{Gc})_{1}+(\mathrm{Man})_{3}(\mathrm{GlcNAc})_{2}$ & Hybrid & CFG \\
\hline (a) 8 & 1927.69301 & 1785.62974 & $(\mathrm{Hex})_{1}(\mathrm{HexNAc})_{2}(\mathrm{Neu} 5 \mathrm{Gc})_{1}+(\mathrm{Man})_{3}(\mathrm{GlcNAc})_{2}$ & Complex & CFG \\
\hline (b) 9 & 2029.7247 & 1873.64578 & $\begin{array}{l}(\mathrm{Hex})_{1}(\mathrm{HexNAc})_{1}(\mathrm{Neu} 5 \mathrm{Ac})_{1}(\mathrm{Neu} 5 \mathrm{Gc})_{1}+(\mathrm{Man})_{3}(\mathrm{Gl}- \\
\mathrm{cNAc})_{2}\end{array}$ & Hybrid & CFG \\
\hline (a) 10 & 2048.71929 & 1906.65602 & $(\mathrm{Hex})_{3}(\mathrm{HexNAc})_{1}(\mathrm{Neu} 5 \mathrm{Gc})_{1}+(\mathrm{Man})_{3}(\mathrm{GlcNAc})_{2}$ & Hybrid & CFG \\
\hline (a) 11 & 2089.74584 & 1947.68257 & $(\mathrm{Hex})_{2}(\mathrm{HexNAc})_{2}(\mathrm{Neu} 5 \mathrm{Gc})_{1}+(\mathrm{Man})_{3}(\mathrm{GlcNAc})_{2}$ & Complex & UniCarbKB \\
\hline 12 & 2175.78261 & 2019.70369 & I.S: $(\mathrm{Hex})_{2}(\mathrm{HexNAc})_{2}(\mathrm{Neu} 5 \mathrm{Ac})_{2}+(\mathrm{Man})_{3}(\mathrm{GlcNAc})_{1}$ & & \\
\hline (d) 13 & 2378.86199 & 2222.78307 & $(\mathrm{Hex})_{2}(\mathrm{HexNAc})_{2}(\mathrm{Neu} 5 \mathrm{Ac})_{2}+(\mathrm{Man})_{3}(\mathrm{GlcNAc})_{2}$ & Complex & UniCarbKB \\
\hline (e) 14 & 2425.88788 & 2297.84026 & $(\mathrm{Hex})_{3}(\mathrm{HexNAc})_{3}(\text { Deoxyhexose })_{2}+(\mathrm{Man})_{3}(\mathrm{GlcNAc})_{2}$ & Complex & UniCarbKB \\
\hline (c) 15 & 3112.12608 & 2970.06281 & $\begin{array}{l}(\mathrm{Hex})_{5}(\mathrm{HexNAc})_{4}(\text { Deoxyhexose })_{1}(\mathrm{Neu} 5 \mathrm{Ac})_{1}+(\mathrm{Man}) 3 \\
(\mathrm{GlcNAc})_{2}\end{array}$ & Complex & UniCarbKB \\
\hline $\begin{array}{l}\text { (e) } 16 \\
\text { (a) } N \text {-glyca }\end{array}$ & 3140.15738 & 3012.10976 & 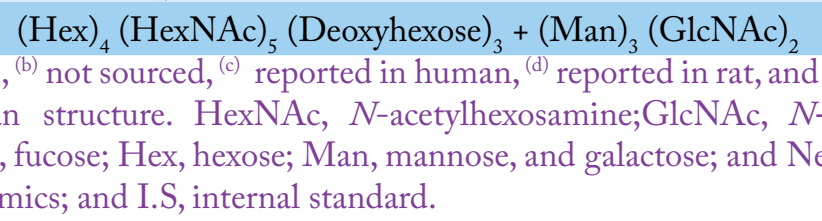 & $\begin{array}{l}\text { Complex } \\
\text { not categorized. } \mathrm{Pe}\end{array}$ & CFG \\
\hline
\end{tabular}

$P=0.006, F_{(3,60)}=38.525, P=0.002$, and $F_{(3,60)}=6.114, P=0.005$, respectively. $\mathrm{GH}-\mathrm{C} 57 \mathrm{BL} / 6$ mice had shown elevation only in two peaks, peaks 13 and 16, when compared to GH$\left.\left.\mathrm{DBA} / 2, F_{(3,60)}=38.525, P=0.007\right), F_{(3,60)}=3.608, P=0.023\right)$, respectively. For the housing condition effect, it was very clear to notice that single housing, especially for DBA/2 caused a dramatic elevation in the majority of our $N$-glycan peaks- peak $4,7,10,13,14,15,16$ when compared to group-housed mice of the same strain, $F_{(3,60)}=1.822$, $P=0.009, \quad F_{(3,60)}=4.862, P=0.033, F_{(3,60)}=5.238, P=0.012$, $F_{(3,60)}=38.525, P=0.002, F_{(3,60)}=6.874, P=0.015, F_{(3,60)}=6.114$, $P=0.011$, and $F_{(3,60)}=3.608, P=0.023$, respectively. GH$\mathrm{C} 57 \mathrm{BL} / 6$ have the only elevation in one peak (peak 13) when compared to $\left.\mathrm{SH}-\mathrm{C} 57 \mathrm{BL} / 6, F_{(3,60)}=38.525, P=0.025\right)$. Also, for the strain*housing condition interaction effect, it was very noticeable that $\mathrm{DBA} / 2$ was very responsive for changes of $N$-glycans, where SH-DBA/2 had a clear elevation for peaks $4,10,14,15$ when compared to $\mathrm{GH}$ $\mathrm{C} 57 \mathrm{BL} / 6,\left(F_{(3,60)}=1.822, P=0.013\right),\left(F_{(3,60)}=4.862, P=0.007\right)$, $\left(F_{(3,60)}=6.874, P=0.007\right)$, and $\left(F_{(3,60)}=6.114, P=0.017\right)$, respectively. Also, separated MS spectra showed $N$-glycans of all experimental groups in Supporting Materials, Suplementry Figure 1. So, we can summarize that the more anxious DBA/2 strain, especially in the single housing condition, had a more responsive elevation in the majority peaks of our estimated $N$-glycan except for peaks 13 and 16.

\section{Glycotyping AnALysis}

In our work, as shown in Table 3, we analyzed our peaks (from peak 1 to peak 16 collectively) to see if we got changes in the complexity of $N$-glycan sugars resulting from the strain and housing condition effects, and we found that constitutional sugars of $\mathrm{N}$-glycan had been modified between groups. For instance, a strain effect was seen where

$\mathrm{SH}-\mathrm{C} 57 \mathrm{BL} / 6$ had more mannose when compared to $\mathrm{SH}-$ $\mathrm{DBA} / 2,\left(F_{(3,60)}=5.014, P=0.001\right)$. Also, GH-C57BL/6 had more tri-fuc and bisecting when compared to GH-DBA/2, $\left(F_{(3,60)}=3.305, P=0.03\right),\left(F_{(3,60)}=2.041, P=0.037\right)$, respectively. In addition, $\mathrm{SH}-\mathrm{DBA} / 2$ had more sial-1 than $\mathrm{SH}-$ C57BL/6, $\left(F_{(3,60)}=2.659, P=0.012\right)$. For the housing condition effect, it was noticeable that SH-DBA/2 had more mono-fuc and bisecting when compared to GH-DBA/2, $\left(F_{(3,60)}=8.527, P=0.002\right),\left(F_{(3,60)}=2.041, P=0.048\right)$, respectively. For strain*housing condition interaction effect, we noticed that $\mathrm{SH}-\mathrm{C} 57 \mathrm{BL} / 6$ had more mannose, mono-fuc, di-fuc, and tri-anten than GH-DBA/2, $\left(F_{(3,60)}=5.041\right.$, $P=0.006),\left(F_{(3,60)}=8.527, P=0.0001\right),\left(F_{(3,60)}=2.105, P=0.03\right)$, $\left(F_{(3,60)}=2.12, \stackrel{P}{P}=0.03\right)$, respectively. Also, GH-C57BL/6 had more tri-fuc than SH-DBA $/ 2,\left(F_{(3,60)}=3.305, P=0.03\right)$. Moreover, SH-DBA/2 had more sial-1 than GH$\mathrm{C} 57 \mathrm{BL} / 6,\left(F_{(3,60)}=2.659, P=0.012\right)$. 
Table 2: $N$-glycan $(\mu \mathrm{M})$ Candidates of C57BL/6 and DBA/2 Mouse Serum, Detected by Highest Amount $(\mathrm{m} \pm \mathrm{SEM})$ and their Significance Levels.

\begin{tabular}{|c|c|c|c|c|c|c|}
\hline \multirow{2}{*}{$\begin{array}{l}\text { Peak } \\
\text { number }\end{array}$} & \multirow[t]{2}{*}{$\mathbf{m} / \mathbf{z}$} & \multirow[t]{2}{*}{ B6GH } & \multirow[t]{2}{*}{ D2GH } & \multirow[t]{2}{*}{ B6SH } & \multirow[t]{2}{*}{ D2SH } & \\
\hline & & & & & & $\begin{array}{l}\text { Significance among groups } \\
\text { and (P-value) }\end{array}$ \\
\hline 1 & 1362.48109 & $1.599688 \pm 0.358$ & $1.644062 \pm 0.264$ & $1.859943 \pm 0.026$ & $1.51222 \pm 0.486$ & -------- ～～～～～～ \\
\hline 2 & 1524.53392 & $2.449914 \pm 0.341$ & $2.735487 \pm 0.303$ & $3.114974 \pm 0.148$ & $2.48021 \pm 0.761$ & -------- \\
\hline 3 & 1590.5921 & $0.60511 \pm 0.268$ & $0.552507 \pm 0.069$ & $0.874316 \pm 0.385$ & $0.453037 \pm 0.148$ & -------- \\
\hline \multirow[t]{3}{*}{4} & \multirow{3}{*}{1724.61363} & \multirow{3}{*}{$1.255782 \pm 0.119$} & \multirow{3}{*}{$1.268822 \pm 0.757$} & \multirow{3}{*}{$1.257644 \pm 0.085$} & \multirow{3}{*}{$1.41012 \pm 0.502$} & ${ }^{(\mathrm{a})} \mathrm{D} 2 \mathrm{SH}>\mathrm{B} 6 \mathrm{SH} \quad\left(0.006^{*}\right)$ \\
\hline & & & & & & b) $\mathrm{D} 2 \mathrm{SH}>\mathrm{B} 6 \mathrm{GH}\left(0.013^{*}\right)$ \\
\hline & & & & & & (c) $\mathrm{D} 2 \mathrm{SH}>\mathrm{D} 2 \mathrm{GH}\left(0.009^{* *}\right)$ \\
\hline 5 & 1752.64493 & $0.676181 \pm 0.123$ & $0.538635 \pm 0.130$ & $0.737794 \pm 0.204$ & $0.691992 \pm 0.021$ & ------------ ～～～～～ \\
\hline 6 & 1870.67154 & $5.19174 \pm 0.552$ & $6.938155 \pm 1.921$ & $5.13491 \pm 1.822$ & $6.358571 \pm 3.394$ & ------------ \\
\hline \multirow[t]{2}{*}{7} & \multirow[t]{2}{*}{1886.66646} & \multirow[t]{2}{*}{$2.660618 \pm 0.237$} & \multirow[t]{2}{*}{$2.988345 \pm 0.430$} & \multirow[t]{2}{*}{$2.725851 \pm 0.329$} & \multirow[t]{2}{*}{$3.427845 \pm 1.173$} & $\begin{array}{l}{ }^{(b)} \mathrm{D} 2 \mathrm{SH}>\mathrm{B} 6 \mathrm{GH} / \mathrm{SH} \\
\left(0.009^{* \prime}\right)\end{array}$ \\
\hline & & & & & & c) $\mathrm{D} 2 \mathrm{SH}>\mathrm{D} 2 \mathrm{GH}\left(0.033^{*}\right)$ \\
\hline 8 & 1927.69301 & $2.678752 \pm 0.710$ & $3.248452 \pm 0.330$ & $3.416554 \pm 0.117$ & $3.252693 \pm 1.023$ & ----------- \\
\hline 9 & 2029.7247 & $7.419128 \pm 0.720$ & $7.220807 \pm 1.549$ & $5.85523 \pm 1.410$ & $8.423314 \pm 4.280$ & ${ }^{(\mathrm{a})} \mathrm{D} 2 \mathrm{SH}>\mathrm{B} 6 \mathrm{SH}(0.016 *)$ \\
\hline \multirow[t]{3}{*}{10} & \multirow[t]{3}{*}{2048.71929} & \multirow[t]{3}{*}{$1.88798 \pm 0.155$} & \multirow[t]{3}{*}{$1.972627 \pm 0.271$} & \multirow[t]{3}{*}{$1.903098 \pm 0.221$} & \multirow[t]{3}{*}{$2.396446 \pm 0.794$} & ${ }^{(a)} \mathrm{D} 2 \mathrm{SH}>\mathrm{B} 6 \mathrm{SH}\left(0.006^{* *}\right)$ \\
\hline & & & & & & (b) $\mathrm{D} 2 \mathrm{SH}>\mathrm{B} 6 \mathrm{GH}\left(0.007^{\prime \prime \prime}\right)$ \\
\hline & & & & & & ${ }^{(\mathrm{c})} \mathrm{D} 2 \mathrm{SH}>\mathrm{D} 2 \mathrm{GH}\left(0.012^{*}\right)$ \\
\hline 11 & 2089.74584 & $14.04291 \pm 3.272$ & $13.95085 \pm 2.200$ & $15.17057 \pm 1.965$ & $15.71592 \pm 6.732$ & ------------ ～～～～～ \\
\hline 12 & 2175.78261 & I.S & & & & \\
\hline \multirow[t]{4}{*}{13} & \multirow[t]{4}{*}{2378.86199} & \multirow{4}{*}{$3.874986 \pm 0.498$} & \multirow[t]{4}{*}{$2.289706 \pm 0.235$} & \multirow[t]{4}{*}{$2.80644 \pm 0.566$} & \multirow[t]{4}{*}{$4.510598 \pm 1.136$} & ${ }^{(\mathrm{a})} \mathrm{D} 2 \mathrm{SH}>\mathrm{B} 6 \mathrm{SH}\left(0.007^{* *}\right)$ \\
\hline & & & & & & a) $\mathrm{B} 6 \mathrm{GH}>\mathrm{D} 2 \mathrm{GH}\left(0.007^{* \prime}\right)$ \\
\hline & & & & & & (c) $\mathrm{D} 2 \mathrm{SH}>\mathrm{D} 2 \mathrm{GH}\left(0.002^{* \prime}\right)$ \\
\hline & & & & & & (c) $\mathrm{B} 6 \mathrm{GH}>\mathrm{B} 6 \mathrm{SH}\left(0.025^{* \prime \prime}\right)$ \\
\hline \multirow[t]{2}{*}{14} & 2425.88788 & $16.58407 \pm 1.785$ & $17.22071 \pm 0.290$ & $20.78292 \pm 2.745$ & $24.63496 \pm 10.215$ & (b) $\mathrm{D} 2 \mathrm{SH}>\mathrm{B} 6 \mathrm{GH}\left(0.007^{* *}\right)$ \\
\hline & & & & & & (c) $\mathrm{D} 2 \mathrm{SH}>\mathrm{D} 2 \mathrm{GH}\left(0.015^{*}\right)$ \\
\hline 15 & 3112.12608 & $2.024847 \pm 0.522$ & $1.754002 \pm 0.240$ & $1.58869 \pm 0.454$ & $2.686581 \pm 1.411$ & ${ }^{(\mathrm{a})} \mathrm{D} 2 \mathrm{SH}>\mathrm{B} 6 \mathrm{SH}\left(0.005^{* *}\right)$ \\
\hline & & & & & & (b) $\mathrm{D} 2 \mathrm{SH}>\mathrm{B} 6 \mathrm{GH}\left(0.017^{\prime \prime}\right)$ \\
\hline & & & & & & (c) $\mathrm{D} 2 \mathrm{SH}>\mathrm{D} 2 \mathrm{GH}\left(0.011^{*}\right)$ \\
\hline 16 & 3140.15738 & $1.854932 \pm 0.618$ & $1.165976 \pm 0.195$ & $1.496282 \pm 0.397$ & $1.552452 \pm 0.756$ & $\begin{array}{l}\text { (a) } \mathrm{B} 6 \mathrm{GH}>\mathrm{D} 2 \mathrm{GH}\left(0.023^{*}\right) \text {, } \\
\text { (c) } \mathrm{D} 2 \mathrm{SH}>\mathrm{D} 2 \mathrm{GH}\left(0.023^{*}\right)\end{array}$ \\
\hline
\end{tabular}

The statistical analysis revealed the highest expression of serum $\mathrm{N}$-glycoforms of mouse, appeared in the general linear model, SPSS."****" showed a significance level, $P \leq 0.001$,"**" a significance level, $P \leq 0.01$,"**” a significance level, $P \leq 0.05$ ); and (---) not detected. B6GH: GH-C57BL/6J; D2GH: GH-DBA/2; B6SH: SH-C57BL/6J; D2SH: SH-DBA/2; I.S., internal standard. (a): strain effect; ${ }^{(b)}$ : strain and housing effect; ${ }^{(c)}$ : housing effect.

\section{Correlation Analysis}

The glycomic analysis of experimental mice showed a novel $\mathrm{N}$-glycomic mechanical pathway and significantly correlated with the animal behavioral findings (Table 4) by a Pearson coefficient correlation test for the duration of grooming and by a Spearman's rho correlation test for grooming bouts. In which, the $N$-glycan in peak7 showed moderate negative correlation with time light (\%) in DL $(r=-0.463, P=0.007)$. Also, peak 10,13, showed moderate negative correlation with grooming bout frequency $(r=-$ $0.463, P=0.008),(r=-0.404, P=0.022)$ respectively, and with time light (\%) in DL ( $r=-0.482, P=0.005),(r=-0.398$, $P=0.024)$, respectively; however positive correlation has been recorded with latency in DL $(r=0.42, P=0.017)$ $(r=0.427, P=0.015)$.Additionally, peak number 14 at $2425 \mathrm{~m} / z$ showed a moderate negative correlation with grooming bout frequency $(r=-0.424, P=0.016)$; however, a positive correlation was recorded with latency in $\mathrm{DL}$ $(r=0.358, P=0.044)$. Lastly, peak number 15 at $3112 \mathrm{~m} / z$ showed a moderate negative correlation with time light $(\%)$ in DL $(r=-0.428, P=0.014)$. 
Table 3: Glycotyping Analyses ( $m \pm$ SEM) of $N$-glycan of C57BL/6 and DBA/2 Mouse Serum.

\begin{tabular}{|c|c|c|c|c|c|}
\hline Glycotyping & B6GH & D2GH & B6SH & D2SH & Significance, $\mathbf{P}$-values \\
\hline \multirow[t]{2}{*}{ High-Man } & \multirow[t]{2}{*}{$3.95 \pm 1.36$} & \multirow[t]{2}{*}{$4.33 \pm 1.53$} & \multirow[t]{2}{*}{$4.97 \pm 1.76$} & \multirow[t]{2}{*}{$3.71 \pm 1.31$} & (a) $\mathrm{B} 6 \mathrm{SH}>\mathrm{D} 2 \mathrm{SH},\left(0.001^{+* *}\right)$ \\
\hline & & & & & (c) $\mathrm{B} 6 \mathrm{SH}>\mathrm{B} 6 \mathrm{GH},\left(0.006^{* *}\right)$ \\
\hline \multirow[t]{2}{*}{ Mono-Fuc } & \multirow[t]{2}{*}{$9.60 \pm 3.62$} & \multirow[t]{2}{*}{$3.27 \pm 1.16$} & \multirow[t]{2}{*}{$20.78 \pm 7.35$} & \multirow[t]{2}{*}{$16.30 \pm 5.76$} & ${ }^{(b)} \mathrm{B} 6 \mathrm{SH}>\mathrm{D} 2 \mathrm{GH},\left(0.0001^{+* \prime}\right)$ \\
\hline & & & & & ${ }^{(c)} \mathrm{D} 2 \mathrm{SH}>\mathrm{D} 2 \mathrm{GH},\left(0.002^{* \prime}\right)$ \\
\hline Di-Fuc & $12.30 \pm 4.47$ & $10.79 \pm 3.81$ & $20.78 \pm 7.35$ & $16.30 \pm 5.76$ & (b) $\mathrm{B} 6 \mathrm{SH}>\mathrm{D} 2 \mathrm{GH},\left(0.030^{*}\right)$ \\
\hline \multirow[t]{2}{*}{ Tri-Fuc } & \multirow{2}{*}{$1.87 \pm 0.64$} & \multirow{2}{*}{$1.20 \pm 0.42$} & \multirow{2}{*}{$1.50 \pm 0.53$} & \multirow{2}{*}{$1.35 \pm 0.48$} & (a) $\mathrm{B} 6 \mathrm{GH}>\mathrm{D} 2 \mathrm{GH},\left(0.005^{* *}\right)$ \\
\hline & & & & & (b) $\mathrm{B} 6 \mathrm{GH}>\mathrm{D} 2 \mathrm{SH},\left(0.030^{*}\right)$ \\
\hline \multirow[t]{2}{*}{ Sial-1 } & \multirow[t]{2}{*}{$29.43 \pm 10.47$} & \multirow[t]{2}{*}{$32.60 \pm 11.52$} & \multirow[t]{2}{*}{$31.20 \pm 11.03$} & \multirow[t]{2}{*}{$37.69 \pm 13.33$} & ${ }^{(a)} \mathrm{D} 2 \mathrm{SH}>\mathrm{B} 6 \mathrm{SH},\left(0.012^{*}\right)$ \\
\hline & & & & & (b) $\mathrm{D} 2 \mathrm{SH}>\mathrm{B} 6 \mathrm{GH},\left(0.044^{\prime \prime}\right)$ \\
\hline Sial-2 & $10.76 \pm 3.80$ & $9.68 \pm 3.42$ & $8.66 \pm 3.06$ & $9.40 \pm 3.33$ & ------------ ～～～～～～ \\
\hline \multirow[t]{2}{*}{ Bisecting } & \multirow[t]{2}{*}{$12.45 \pm 4.52$} & \multirow[t]{2}{*}{$2.67 \pm 0.94$} & \multirow[t]{2}{*}{$10.31 \pm 3.65$} & \multirow[t]{2}{*}{$13.00 \pm 4.60$} & ${ }^{(\mathrm{c})} \mathrm{D} 2 \mathrm{SH}>\mathrm{D} 2 \mathrm{GH},\left(0.048^{*}\right)$ \\
\hline & & & & & ${ }^{(\mathrm{a})} \mathrm{B} 6 \mathrm{GH}>\mathrm{D} 2 \mathrm{GH},\left(0.037^{\prime \prime}\right)$ \\
\hline Bi-anten & $20.94 \pm 7.52$ & $20.58 \pm 7.37$ & $23.01 \pm 8.13$ & $19.66 \pm 6.95$ & ------------ \\
\hline Tri-anten & $12.30 \pm 4.47$ & $10.79 \pm 3.81$ & $20.78 \pm 7.35$ & $16.30 \pm 5.76$ & (b) $\mathrm{B} 6 \mathrm{SH}>\mathrm{D} 2 \mathrm{GH},\left(0.030^{*}\right)$ \\
\hline Tetra-anten & $3.99 \pm 1.37$ & $2.98 \pm 1.05$ & $3.08 \pm 1.09$ & $3.67 \pm 1.30$ & ${ }^{(\mathrm{a})} \mathrm{B} 6 \mathrm{GH}>\mathrm{D} 2 \mathrm{GH},(0.081)$ \\
\hline \multicolumn{6}{|c|}{ 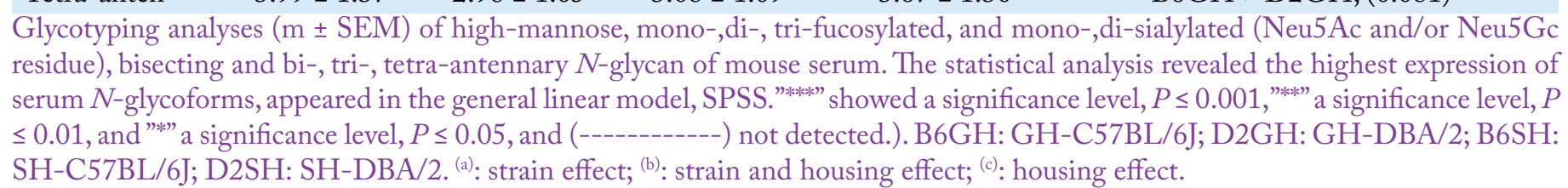 } \\
\hline
\end{tabular}

Table 4: The Correlations Between Grooming Frequency, Time Light (\%), and Latency in DL with Serum $N$-glycan Peaks of C57BL/6 and DBA/2 Mouse.

\begin{tabular}{|c|c|c|c|c|c|c|c|c|c|c|}
\hline \multicolumn{11}{|l|}{$\mathrm{N}$-glycan peaks } \\
\hline Correlations Coefficient & $\begin{array}{l}\text { Peak } \\
\text { No. } 1\end{array}$ & $\begin{array}{l}\text { Peak } \\
\text { No. } 2\end{array}$ & $\begin{array}{l}\text { Peak } \\
\text { No. } 4\end{array}$ & $\begin{array}{l}\text { Peak } \\
\text { No. } 7\end{array}$ & $\begin{array}{l}\text { Peak } \\
\text { No. } 9\end{array}$ & $\begin{array}{l}\text { Peak } \\
\text { No. } 10\end{array}$ & $\begin{array}{l}\text { Peak } \\
\text { No. } 13\end{array}$ & $\begin{array}{l}\text { Peak } \\
\text { No. } 14\end{array}$ & $\begin{array}{l}\text { Peak } \\
\text { No. } 15\end{array}$ & $\begin{array}{l}\text { Peak } \\
\text { No. } 16\end{array}$ \\
\hline Significance (Grooming bouts) & 0.058 & 0.015 & -0.255 & -0.295 & -0.143 & -0.463 & -0.404 & -0.424 & -0.252 & -0.066 \\
\hline Spearman's rho (r) & 0.752 & 0.934 & 0.159 & 0.101 & 0.435 & 0.008 & 0.022 & 0.016 & 0.164 & 0.721 \\
\hline Significance (Time light (\%) in DL) & -0.121 & -0.12 & -0.34 & -0.358 & -80 & -0.482 & -0.398 & -0.315 & -0.117 & 0.019 \\
\hline PearsonCorrelation(r) & 0.51 & 0.512 & 0.057 & 0.044 & 0.665 & 0.005 & 0.024 & 0.079 & 0.523 & 0.918 \\
\hline Significance (Latency in DL) & -0.168 & -0.085 & 0.163 & 0.244 & 0.089 & 0.42 & 0.427 & 0.358 & 0.099 & -0.015 \\
\hline Pearson Correlation(r) & 0.359 & 0.645 & 0.371 & 0.179 & 0.627 & 0.017 & 0.015 & 0.044 & 0.591 & 0.936 \\
\hline
\end{tabular}

Each value represented Spearman's rho correlation coefficients and Pearson Correlation $(r)=0.3-0.5$, weak to moderate correlation. DL: dark light; No.: Number.

\section{ANXIETY-RELATED BEHAVIORS IN DBA/2 AND C57BL/6J MICE}

Home cage behavioral differences between C57BL6 and DBA/2 mice in the two housing conditions were initially reported in our previous published paper (Abou-Elnaga et al., 2019), where a detailed behavioral ethogram was used to evaluate their anxiety levels based on their home-cage behaviors (interaction with enriched environment, grooming, bedding-directed behavior, feeding, sleep, exploratory behavior, and locomotion). Our previous finding agreed with many authors who stated that DBA/2 mice are more anxious than C57BL/6J (Võikar et al., 2005; Brigman et al., 2009). The results of the detailed behavioral analyses for both mice strains (C57BL/6J and DBA/2) in single versus group housing (Abou-Elnaga et al., 2019) are added in the Suplementry Table 1 of the Supporting Information. However, other studies were comparing anxiety-related behavior by various anxiety tests that had no consistency in their results. DBA/2 mice were less anxious and displayed higher activity in the light area of the DL test (Beuzen and Belzung, 1995), but DBA/2 exhibited higher anxiety-like behavior than the C57BL/6 mice in the DL test in a study by Eltokhi et al. (2020). Moreover, DBA/2 mice were less anxious in the elevated plus-maze than C57BL/6 (Eltokhi et al., 2020), whereas DBA/2 was more anxious in the same test in Võikar et al. (2005).

To help eliminate confusion, anxiety-related behavior 
merited more approaches to assess the differences between both strains. A familiar way to evaluate anxiety is using the induced grooming test and DL test to assess gross activity characteristics such as the latency to groom, frequency, duration of grooming, percent of the time in the light, and latency to enter the light compartment (Eltokhi et al., 2020). It was reported that using grooming measurements can be used for quantifying obsessive-compulsive disorder (OCD) and Tourette syndrome severity in the mouse model (Ahmari et al., 2016). Consistent with this, psychotropic drugs that exacerbate OCD in clinical patients have been reported to elicit robust grooming in rodents (Zike et al., 2017). Therefore, the current study focused on detailed analyses of anxiety-related behaviors in induced grooming test and DL exploration test in single versus group housing conditions of $\mathrm{DBA} / 2$ and $\mathrm{C} 57 \mathrm{BL} / 6 \mathrm{~J}$ mice.

Our findings showed that mice from $\mathrm{DBA} / 2$ and C57BL/6J mice strains exhibited divergent behavioral patterns. Overall, DBA/2 mice had grooming activity, lower vertical activity, higher levels of defecation, more latency to begin rearing and grooming, less time in light, and a higher latency to enter the light compartment in the DL test. From here, we can hypothesize the following: lower grooming levels in DBA/2 mice may result from lower activity levels in DBA/2 when compared to the C57BL/6J strain (Abou-Elnaga et al., 2019). Additionally, grooming has been identified as a behavioral pattern of stress in rodents raising the possibility that less grooming in DBA/2 mice may reflect a higher anxiety level (Abou-Elnaga et al., 2019), which would agree with a lot of studies stating that grooming may be inhibited in stressful conditions and during anxiety due to its effect on locomotion activity and the occurrence of the freezing response (Kalueff et al., 2016). A significant decrease in the grooming frequency and duration in single housed C57BL/6J and DBA/2 mice may result from the stressful condition of a single housing. However, such reduction in grooming activity appeared with high significance in C57BL/6J mice; it may have occurred because the C57BL/6Jmouse is genetically known to be more active compared to the DBA/2 mouse strain, and consequently groomed more. Therefore, the significant effect of single housing was more obvious in C57BL/6 mice because they performed more time in grooming activities than DBA/2 (Hatcher et al., 2001).

Our data also indicated that less rearing, and more latency to rear in DBA/2 mice. Vertical rearing is the main parameter that can be used to assess general physical-motor abilities, level of interest towards a novel environment, and anxiety (Pitsikas et al., 2018).Therefore, we suggest that low rearing may indicate more anxiety in DBA/2. Interestingly, our result agreed with authors who postulated that decreased rearing behavior was indicative of increased anxiety level in DBA/2 mice (Seibenhener and Wooten,
2015). In the same point of view, defecation and urination can be used as measures of individual differences in emotions that more anxious mice had a higher level of defecation in different behavioral tests than the less anxious ones (Lavu et al., 2020). Noticeably, for strain effect, DBA/2 showed more anxiety than the C57BL/6 mice, whereas for the housing condition effect it was obvious that anxiety was exaggerated in the single-housed mice in the DL exploration test only for the DBA/2 model. DBA/2 mice were more affected by housing conditions. This seems to agree with previous data suggesting that there was a different reaction to the environmental manipulations between the $\mathrm{C} 57 \mathrm{BL} / 6$ and the DBA/2mice and housing-dependent differences were more evident in the $\mathrm{DBA} / 2$ strain (Võikar et al., 2005).

\section{Functional Glycomics In DBA/2 And C57BL/6 JMICE}

The selected $15 \mathrm{~N}$-glycans structures in mice sera were identified in Glycosuit DB and CFG, and also shown in previous reports (Mahmoud et al., 2019; Rehan et al., 2020; Gizaw et al., 2015; Gizaw et al., 2016; Gebrehiwot et al., 2018; Rehan et al.,2015). Our results demonstrated that the mice sera contained both Neu5Ac and Neu5Gc-sial-terminal(s) due to the effect of the CMP-Neu5Ac Hydroxylase enzyme that converted Neu5Ac to Neu5Gc (Varki, 2001; Varki, 2007; Mahmoud et al., 2019; Rehan et al., 2020). In a previously published paper (Mahmoud et al., 2019; Rehan et al., 2020), we illustrated that sialylated $N$-glycans (Neu5Ac-terminals; peak 13 "2 X Neu5Ac-terminals" and peak 15 " $1 \mathrm{X}$ Neu5Ac-terminal") regulate the homeostatic immune balance of mice. Also, the serum $N$-glycan peak at $2410 \mathrm{~m} / z$ (2X Neu5Gc-terminals) was highly expressed as a biomarker of chronic stress in BALB/c mice (Mahmoud et al., 2019) or in mice infected with acute/chronic Toxoplasma gondii (Rehan et al., 2020). In our previous research, expressing the peak $2410 \mathrm{~m} / \mathrm{z}$ is evidence of an immune compensatory mechanism of (stressed/infected) $\mathrm{BALB} / \mathrm{c}$ mice to cope with the depressive-like challenge (Mahmoud et al., 2019; Rehan et al., 2020).

Interestingly, peaks of the mouse $\mathrm{N}$-glycan number 4,7 , $8,9,10,11,13$, and 15 at $1724 \mathrm{~m} / z, 1886 \mathrm{~m} / z, 1927 \mathrm{~m} / z$, $2029 \mathrm{~m} / z, 2048 \mathrm{~m} / \mathrm{z}, 2089 \mathrm{~m} / \mathrm{z}, 2378 \mathrm{~m} / z$, and $3112 \mathrm{~m} / \mathrm{z}$, were significantly elevated in $\mathrm{DBA} / 2$ mice, especially in single housing condition when compared to the other groups, giving us a theory for mice's anxiety responses by post-translational protein glycosylation mechanism. Our findings are supported by previous research that reported that sialylated $N$-glycans are significantly altered in diseased/stressed patients compared to the control subjects (Saldova et al., 2010; Terashima et al., 2014). Interestingly, it was clear that during the $N$-glycome pathway from peak 1 to peak 16 the expression levels of $N$-glycans of Neu5Gc terminal (in peak $4,7,9,10,11$ ) were dramatically increased 
in SH-DBA/2 mice, which we hypothesize is a response to the anxiety state by modifying $N$-glycome profiles.

Also, we got more significant expressions of $N$-glycans of Neu5Acs (inpeak13 " 2 X Neu5Ac" and peak15 " $1 \mathrm{X} \mathrm{Neu-}$ $5 \mathrm{Ac}$ ") instead of Neu5Gcs, which confirmed the anxiety challenge and the immune defense in vivo, such modification of Neu5Gc into Neu5Ac terminal (shown in peaks13, 15) are peaks supposed to be a compensatory mechanism for anxiety state (Mahmoud et al., 2019; Rehan et al., 2020; Gizaw et al., 2015; Gizaw et al., 2016; Gebrehiwot et al., 2018; Rehan et al., 2015; Reiding et al., 2016). In addition, peaks 10, 13 (Neu5Gc and Neu5Ac terminals) showed a moderate negative correlation with grooming bouts frequency and with percent time in the light compartment in DL, also peak 14 (multi-antennary structures of $N$-glycan) showed the same correlation with grooming bouts frequency and a moderate negative correlation was noticed with latency to enter the light compartment in DL. The modest correlation is realistic because it might relate to the influences of anxiety-adapted responses of experimental mice on the biosynthetic pathway of $N$-glycome profiles through the epigenetic background on genetic DNA-mutations, along with the possible roles of sialyltransferases and glycosyltransferases enzymes. Altogether, would cope, as possible, the anxiety threshold for regulating the homeostasis and for making a compensatory mechanism, particularly in DBA/2 strain.

In contrast, peaknumber16 (tetra-antennary, and bisecting $\mathrm{N}$-glycan) was significantly expressed in $\mathrm{GH}-\mathrm{C} 57 \mathrm{BL} / 6 \mathrm{~J}$, which indicated the smoother sequences of sugars to the glycan structures due to enzymatic activity and $\mathrm{N}$-glycan reaching the natural end of its pathway. The biosynthetic pathway of sialylated $\mathrm{N}$-glycans, Neu5Ac/Neu5Gc-terminals, significantly confirmed the evidence of glycosyltransferase enzymes for maintaining homeostasis; particularly in $\mathrm{SH}-\mathrm{DBA} / 2$ mice. However, the different expression levels of $N$-glycans seemed likely to be associated with anxiety, which might influence the sialyltransferase and sialidase enzymes' activities (Mahmoud et al., 2019; Rehan et al., 2020; Gebrehiwot et al., 2018). From our current results, we find that the biosynthetic pathway of $N$-glycome is influenced by the anxiety state and correlated to behavioral results of mice, so we hypothesize that sialylated $N$-glycomes profile can be used as a biomarker for assessing the level of anxiety in $\mathrm{C} 57 \mathrm{BL} / 6$ and $\mathrm{DBA} / 2$ mice.

Glycotyping analysis revealed that complicated $N$-glycans, such as fucosylated-, sialylated-, and bisecting- $N$-glycans, were more highly expressed in the $\mathrm{DBA} / 2$ than in the C57BL/6 strain, indicating more anxiety levels in DBA/2. Noticeably, the high-mannose/simple $N$-glycans identified in peaks 1 and 2 were of the highest peaks/expressions in C57BL/6J, due to the activity of exo-mannosidases en- zymes responsible for trimming the multi-mannose residues. We found that that the higher activity of exo-mannosidases, the better the fitness condition of $\mathrm{C} 57 \mathrm{BL} / 6$ when compared to the DBA/2 mice. This result was similar to a previous report showing that significant amounts of high-mannose $\mathrm{N}$-glycan structures (mannose $9 \sim$ mannose 5) were detected in Holstein cow sera in the spring season of the optimum weather condition to get the maximum milk production )Rehan et al., 2015 (.Therefore, the MS spectra of $N$-glycomics in mice sera significantly showed the simplicity in $\mathrm{SH}-\mathrm{C} 57 \mathrm{BL} / 6$ while the complexity of $\mathrm{N}$-glycan structures was in SH-DBA/2 mice, respectively, because of the enzymatic activities during the post-translational modifications of glycoproteins under stress condition. Finally, our finding in C57BL/6 and DBA/2 mice may create a significant diagnostic biomarker of anxiety, and therefore we confirm that the behavioral observations and $\mathrm{N}$-glycome profiles collectively can play vital diagnostic markers of emotional behaviors in mice.

\section{CONCLUSIONS}

We provided evidence that measured parameters in induced grooming test and DL detected in C57BL/6 and $\mathrm{DBA} / 2$ mice may be related to higher anxiety of $\mathrm{DBA} / 2$ and a lower anxiety level in C57BL/6 mice. By employing a glycoblotting approach combined with MALDI-TOF/ MS analysis of serum glycoproteins from these mice, we found differences in $N$-glycans peak expression that was correlated with anxiety. This alteration of $N$-glycome expression might come also from housing conditions (single versus group). Consequently, the mice's behavioral adaptations to such stressors made an elevation of the biosynthetic pathway and enzymatic activities of their serum $\mathrm{N}$-glycan structures. We reveal that sialylated $\mathrm{N}$-glycan is a marker of anxiety in C57BL/6 and DBA/2 mice. Therefore, our results indicate that the DBA/2 mice might be of use in neurobiology research as an anxiety-sensitive model animal.

\section{ACKNOWLEDGMENTS}

The authors thank the Faculty of Veterinary Medicine at Mansoura and Menofia universities, Egypt for helping us to finish the work. We deeply appreciate the referees' efforts in the assessment of this article.

\section{AUTHORS' CONTRIBUTIONS}

A.F.A and I.F.R mutually contributed chemicals, materials, and research methods in the manuscript. G.E.B shared in the strategy and sample analysis. All authors took part in the in-vitro as well as in vivo analysis. They drafted, revised, and approved the manuscript. 


\section{CONFLICTS OF INTEREST}

The authors confirmed that there were no conflicts of interest.

\section{SUPPLEMENTARY MATERIALS}

The supplementary material was written in Table S1 and Figure S1. Moreover, more detailed information of chemicals and $N$-glycomic protocol, written in the Supporting Information I, II.

\section{REFERENCES}

-Abou-Elnaga AF, Rehan, IF, Thompson RR, Abou-Ismail UA, Mahmoud ME, Radi AM, Mohammed HH, Sabek, AA, Elhussiny, MZ (2019). The effects of group versus single housing on home cage behaviour in two strains of laboratory mice. Slovenian Veterinary Research. 56: 22, 39-49. https:// doi.org/10.26873/SVR-742-2019

-Ahmari SE. (2016). Using mice to model obsessive compulsive disorder: freom genes to circuits. Neuroscience. 3: 321, 121137. https://doi.org/10.1016/j.neuroscience.2015.11.009

-Beuzen A, Belzung C (1995). Link between emotional memory and anxiety states: a study by principal component analysis. Physiol. Behaviour. 58: 1, 111-118. https://doi. org/10.1016/0031-9384(95)00013-9

-Brigman JL, Ihne J, Saksida LM, Bussey T, Holmes A (2009). Effects of subchronic phencyclidine (PCP) treatment on social behaviors, and operant discrimination and reversal learning in C57BL/6J mice. Front. Behavio. Neurosci. 3:2. https://doi.org/10.3389/neuro.08.002.2009.

-Eltokhi A, Kurpiers B, Pitzer C (2020). Behavioral tests assessing neuropsychiatric phenotypes in adolescent mice reveal strain- and sex-specific effects. Scientific Reports. 10, 11263. https://doi.org/10.1038/s41598-020-67758-0

-Filiou MD, Nussbaumer M, Teplytska L, Christoph W. Turck (2021). Behavioral and metabolome differences between c57b1/6 and $\mathrm{dba} / 2$ mouse strains: implications for their use as models for depression- and anxiety-like phenotypes. Metabolites. 11: 2, 128. https://doi.org/10.3390/ metabo11020128

- Gebrehiwot AG, Melka DS, Kassaye YM, Rehan, IF, Rangappa S, Hinou H, Kamiyama T, Nishimura SI (2018). Healthy human serum $\mathrm{N}$-glycan profiling reveals the influence of ethnic variation on the identified cancer-relevant glycan biomarkers. PloSONE. 13: 12, 020951. https://doi. org/10.1371/journal.pone.0209515

- Gizaw ST, Koda T, Amano M, Kamimura K, Ohashi T, Hinou H, Nishimura SI (2015). A comprehensive glycome profiling of Huntington's disease transgenic mice. Biochimica et Biophysica Acta (BBA)-General Subjects. 1850(9): 17041718. https://doi.org/10.1016/j.bbagen.2015.04.006.

- Gizaw ST, Ohashi T, Tanaka M, Hinou H, Nishimura SI (2016). Glycoblotting method allows for rapid and efficient glycome profiling of human Alzheimer's disease brain, serum and cerebrospinal fluid towards potential biomarker discovery. Biochim. Biophys. Acta (BBA)-General Subjects. 1860:8, 1716-1727. https://doi.org/10.1016/j.bbagen.2016.03.009.

-Hatcher JP, Jones DN, Rogers DC, Hatcher PD, Reavill C, Hagan JJ, Hunter AJ (2001). Development of SHIRPA to characterise the phenotype of gene-targeted mice. Behaviour.
Brain Res. 125: 1-2, 43-47. https://doi.org/10.1016/S01664328(01)00275-3.

- Hirose K, Amano M, Hashimoto R, Lee YC, Nishimura SI (2011). Insight into glycan diversity and evolutionary lineage based on comparative avio- $\mathrm{N}$-glycomics and sialic acid analysis of $88 \mathrm{egg}$ whites of galloanserae. Biochemistry. 50:21, 4757-4774. https://doi.org/10.1021/bi101940x.

- Kalueff AV, Stewart AM, Song C, Berridge KC, Graybiel AM (2016). Neurobiology of rodent self-grooming and its value for translational neuroscience. Nat. Rev. Neuroscience. 17: 1, 45-59. https://doi.org/10.1038/nrn.2015.8

- Kalueff AV, Tuohimaa P (2004). Grooming analysis algorithm for neurobehavioural stress research. Brain Research Protocol. 13: 3, 151-158. https://doi.org/10.1016/j. brainresprot.2004.04.002

• Kamakura R, Kovalainen M, Leppäluoto J, Herzig KH, Mäkelä KA (2016). The effect of group and single housing and automated animal monitoring on urinary corticosterone levels in male C57BL/6 mice. Physiology Reproduction. 4: 3, 12703. https://doi.org/10.14814/phy2.12703

-Jirkof P, Rudeck J, Lewejohanan L (2019). Assessing affective state in laboratory rodents to promote animal welfare- what is the progress in applied refinement research?. Animals. 9, 1026. https://doi.org/10.3390/ani9121026

- Lavu M, Tobias BC, Schuette PJ, Adhikari A (2020). To approach or avoid: an introductory overview of the study of anxiety using rodent assays. Front. Behav. Neurosci. 14:145. https://doi.org/10.3389/fnbeh.2020.00145

- Mahmoud ME, Rehan IF, Ahmed KE, Abdelrahman A, Mohammadi S, Abou-Elnaga AF, Youssef M, Diab HM, Salman D, Elnagar A, Mohammed HH, Shanab O, Ibrahim RM., Ahmed EK, Hesham A, Gupta A (2019).Identification of $\mathrm{N}$-glycoproteins as a biological correlates underlying chronic stress response in mice serum. Mol. Biol. Rep. 46: 3, 2733-2748. https://doi.org/10.1007/s11033-019-04717-7

- Miura Y, Hato M, Shinohara Y, Kuramoto H, Furukawa JI, Kurogochi M, Shimaoka H, Tada M, Nakanishi K, Ozaki M, Todo S (2008). BlotGlycoABC ${ }^{\mathrm{TM}}$, an integrated glycoblotting technique for rapid and large scale clinical glycomics. Molecul. Cellul. Proteom. 7:2, 370-3707. https:// doi.org/10.1074/mcp.M700377-MCP200.

-Mu MD, Geng HY, Rong KL, Peng RC, Wang ST, Geng LT, Qian ZM, Yung WH, Ke Y (2020). A limbic circuitry involved in emotional stress-induced grooming. Nature Communication. 11: 1, 2261. https://doi.org/10.1038/ s41467-020-16203-x

-Pitsikas N, Georgiadou G, Delis F, Antoniou K (2018). Effects of anesthetic ketamine on anxiety-like behaviour in rats. Neurochemical research. 44: 829-838. https://doi. org/10.1007/s11064-018-02715-y

- Rehan IF, Mahmoud ME, Salman D, Elnagar A, Salman S, Youssef M, Abdel Aziz AR, Bazh EK, Hesham A (2020). Sialylated $\mathrm{N}$-glycan profile during acute and chronic Toxoplasma gondii in mice. Sci. Rep-Nat. 10: 1, 3809. https://doi.org/10.1038/s41598-020-60681-4

- Rehan IF, Ueda K, Mitani T, Amano M, Hinou H, Ohashi T, Kondo S, Nishimura SI (2015). Large-scale glycomics of livestock: discovery of highly sensitive serum biomarkers indicating an environmental stress affecting immune responses and productivity of Holstein dairy cows. J. Agric. Food. Chem. 63: 48, 10578-10590. https://doi.org/10.1021/ acs.jafc. 5 b0 04304

- Reiding KR, Ederveen AL, Rombouts Y, Wuhrer M (2016). 
Murine plasma $\mathrm{N}$-glycosylation traits associated with sex and strain. J. Proteome. Res. 15: 10, 3489-3499. https://doi. org/10.1021/acs.jproteome.6b00071

- Saldova R, Fan Y, Fitzpatrick JM, Watson RW, Rudd PM (2011). Core fucosylation and $\alpha 2-3$ sialylation in serum $\mathrm{N}$-glycome is significantly increased in prostate cancer comparing to benign prostate hyperplasia. Glycobiology. 21(2): 195-205. https://doi.org/10.1093/glycob/cwq147.

- Sanes JT, Hinou H, Lee YC, Nishimura SI (2018). Glycoblotting of egg white reveals diverse $\mathrm{N}$-glycan expression in quail species. J. Agric. Food. Chem. 67: 1, 531-540. https://doi. org/10.1021/acs.jafc.8b04782

- Seibenhener ML, Wooten MC (2015). Use of the open field maze to measure locomotor and anxiety-like behavior in mice. J. Vis. Exp. 96: 52434. https://doi.org/10.3791/52434

- Terashima M, Amano M, Onodera T, Nishimura SI, Iwasaki N (2014). Quantitative glycomics monitoring of induced pluripotent-and embryonic stem cells during neuronal differentiation. Stem Cell. Res. 13: 3,454-464. https://doi. org/10.1016/j.scr.2014.10.006

- Varki A (2001). Loss of N-glycolylneuraminic acid in humans: mechanisms, consequences, and implications for hominid evolution. American Journal of Physical Anthropology: The Official Publication of the American Association of Physical Anthropologists.116: S33, 54-69. https://doi.org/10.1002/

\section{SUPPLEMENTARY MATERIAL}

\section{Chemicals And Reagents}

Three-Methyl-1-p-tolyltriazene (MTT), ammonium bicarbonate 99\% (ABC), sodium 2-hydroxy-3-tetradecanamidopropane-1-sulfonate[1-propanesulfonic acid, 2-hydroxyl-3-myristamido, sodium salt (1:1); PHM], disialyloctasaccharide, and O-benzylhydroxylamine hydrochloride (BOA) were obtained from Tokyo Chemical Industry Co., Ltd. (Tokyo, Japan). BlotGlycoH beads were purchased from Sumitomo Bakelite, Co., Ltd. (Tokyo, Japan). Peptide $N$-glycosidase F (PNGase F) was purchased from New England Biolabs Inc. (Ipswich, MA, USA). Trypsin, dithiothreitol (DTT), and iodoacetamide (IAA) were purchased from Sigma-Aldrich, Inc. (St. Louis, MO, USA). SweetBlot was obtained from Systems Instruments Co., Inc. (Hachioji, Japan). MultiScreen Solvinert filter plates $(0.45 \mu \mathrm{m}$ low binding hydrophilic PTFE, MSRLN0410) were purchased from Millipore Co., Inc. (Tokyo, Japan). Mass measurements were performed using MALDI-TOF/MS (Ultraflex III, Bruker Daltonics, Germany), and IMB-SPSS software was obtained from IBM Co., Inc. (Armonk, NY, USA).

\section{Glycoblotting-Based Serum Glycomics}

Pretreatment ( $\mathrm{N}$-glycan realease): The premix composition [0.33 M ABC (2.52 mL), 0.4\% PHM in $10 \mathrm{mMABC}$

ajpa.10018.

-Varki A (2007). Glycan-based interactions involving vertebrate sialic-acid-recognizing proteins. Nature. 446:7139, 10231029. https://doi:10.1038/nature05816.

-Võikar V, Polus A, Vasar E, Rauvala H (2005). Long-term individual housing in $\mathrm{C} 57 \mathrm{BL} / 6 \mathrm{~J}$ and $\mathrm{DBA} / 2$ mice: assessment of behavioral consequences. Genes Brain Behav. 4: 4, 240-252. https://doi.org/10.1111/j.1601183X.2004.00106.x

- Voikar V, Vasar E, Rauvala H (2004). Behavioral alterations induced by repeated testing in C57BL/6J and 129S2/ Sv mice: implications for phenotyping screens. Genes Brain Behav. 3: 1, 27-38. https://doi.org/10.1046/j.1601183X.2003.0044.x

- Yamagata H, Uchida S, Matsuo K, Harada K, Kobayashi A, Nakashima M, Higuchi F, Watanuki T, Matsubara T, Watanabe Y (2018). Altered plasma protein glycosylation in a mouse model of depression and in patients with major depression. J. Affect. Disord. 233: 79-85. https://doi. org/10.1016/j.jad.2017.08.057

-Zike I, Xu T, Hong N, Vanderweele JV (2017). Rodent models of obsessive compulsive disorder: Evaluation validity to interpret emerging neurobiology. Neuroscience.14:345,256273. https://doi.org/10.1016/j.neuroscience.2016.09.012

(1.26 mL), $120 \mathrm{mM}$ DTT (0.84 mL), mess up to $8.4 \mathrm{~mL}$ with water] was prepared, and then $50 \mu \mathrm{L}$ of that premix was freshly added to serum of each mouse $(10 \mu \mathrm{L})$. The internal standard (disialyl-octasaccharide) was added carefully (40 $\mu \mathrm{M}$ final concentration) was added carefully and the mixture was incubated at $60^{\circ} \mathrm{C}$ for $30 \mathrm{~min}$. Freshly prepared $20 \mu$ Lof $123 \mathrm{mM}$ IAA was added and incubated to allow alkylation process at $25^{\circ} \mathrm{C}$ for $60 \mathrm{~min}$ in the dark. The mixture was then treated with Trypsin $(10 \mu \mathrm{L}, 40 \mathrm{U} /$ $\mu \mathrm{L}$ in $1 \mathrm{mM} \mathrm{HCl}$ ) at $37^{\circ} \mathrm{C}$ for 3 hours, followed by heat inactivation of the enzyme at $90^{\circ} \mathrm{C}$ for $10 \mathrm{~min}$ after being cooled to room temperature. $N$-glycans was enzymatically released from trypsin-digested glycopeptides by incubation with 65 units of PNGase $\mathrm{F}$ at $37^{\circ} \mathrm{C}$ overnight. Further, 60 $\mu \mathrm{L}$ of digested mixture containing $N$-glycans was directly applied to glycoblotting technology.

Glycoblotting (chemoselective ligation, washing, on-bead esterifications, and imine exchange): Five hundred microliters of BlotGlyco ${ }^{\oplus} \mathrm{H}$ beads in a $10 \mathrm{mg} / \mathrm{mL}$ suspensionwith water was initially placed into the wells of a MultiScreen Solvinert ${ }^{\oplus} 96-$ well filter plate, and water was then removed by a vacuum. The digested mixtures of mouse serum $(20$ $\mu \mathrm{L}$ ) containing released $N$-glycan were applied to each well together with $180 \mu \mathrm{L}$ of $2 \% \mathrm{AcOH} / \mathrm{CH}_{3} \mathrm{CN}$. 
Table S1: The Detailed Behavioural Ethogram of C57BL6 and DBA/2 Mouse.

\begin{tabular}{|c|c|c|c|c|c|c|}
\hline Behavioural ethogram & B6GH & D2GH & B6SH & D2SH & Results & P-value \\
\hline \multirow[t]{3}{*}{ In crawl-ball } & \multirow[t]{3}{*}{$23.99 \pm 3.52$} & \multirow[t]{3}{*}{$56.14 \pm 4.54$} & \multirow[t]{3}{*}{$54.86 \pm 4.25$} & \multirow[t]{3}{*}{$72.84 \pm 3.56$} & $\mathrm{D} 2 \mathrm{GH}>\mathrm{B} 6 \mathrm{GH}$ & $\mathrm{P}<0.001$ \\
\hline & & & & & $\mathrm{D} 2 \mathrm{SH}>\mathrm{B} 6 \mathrm{SH}$ & $\mathrm{P}<0.001$ \\
\hline & & & & & $\mathrm{B} 6 \mathrm{SH}>\mathrm{B} 6 \mathrm{GH}$ & $\mathrm{P}<0.01$ \\
\hline \multirow[t]{2}{*}{ Home-cage grooming } & \multirow[t]{2}{*}{$9.02 \pm 1.05$} & \multirow[t]{2}{*}{$7.67 \pm 1.14$} & \multirow[t]{2}{*}{$6.80 \pm 1.94$} & \multirow[t]{2}{*}{$11.82 \pm 1.47$} & $\mathrm{D} 2 \mathrm{SH}>\mathrm{D} 2 \mathrm{GH}$ & $\mathrm{P}<0.01$ \\
\hline & & & & & $\mathrm{D} 2 \mathrm{SH}>\mathrm{B} 6 \mathrm{SH}$ & $\mathrm{P}<0.05$ \\
\hline \multirow[t]{2}{*}{ Bedding-directed behaviour } & \multirow{2}{*}{$13.88 \pm 1.22$} & \multirow{2}{*}{$14.93 \pm 1.58$} & \multirow{2}{*}{$22.08 \pm 3.02$} & \multirow[t]{2}{*}{$13.83 \pm 1.15$} & $\mathrm{~B} 6 \mathrm{SH}>\mathrm{D} 2 \mathrm{SH}$ & $\mathrm{P}<0.01$ \\
\hline & & & & & $\mathrm{B} 6 \mathrm{SH}>\mathrm{B} 6 \mathrm{GH}$ & $\mathrm{P}<0.01$ \\
\hline \multirow[t]{2}{*}{ Feeding } & \multirow[t]{2}{*}{$6.07 \pm 0.99$} & \multirow[t]{2}{*}{$13.88 \pm 0.75$} & \multirow[t]{2}{*}{$6.11 \pm 0.86$} & \multirow[t]{2}{*}{$7.32 \pm 0.98$} & $\mathrm{D} 2 \mathrm{GH}>\mathrm{B} 6 \mathrm{GH}$ & $\mathrm{P}<0.001$ \\
\hline & & & & & $\mathrm{D} 2 \mathrm{GH}>\mathrm{D} 2 \mathrm{SH}$ & $\mathrm{P}<0.001$ \\
\hline \multirow[t]{4}{*}{ Sleep } & \multirow[t]{4}{*}{$43.02 \pm 2.75$} & \multirow[t]{4}{*}{$55.10 \pm 3.45$} & \multirow[t]{4}{*}{$37.50 \pm 3.98$} & \multirow[t]{4}{*}{$61.38 \pm 5.12$} & $\mathrm{D} 2 \mathrm{GH}>\mathrm{B} 6 \mathrm{GH}$ & $\mathrm{P}<0.01$ \\
\hline & & & & & $\mathrm{D} 2 \mathrm{GH}>\mathrm{B} 6 \mathrm{SH}$ & $\mathrm{P}<0.001$ \\
\hline & & & & & $\mathrm{D} 2 \mathrm{SH}>\mathrm{B} 6 \mathrm{GH}$ & $\mathrm{P}<0.001$ \\
\hline & & & & & D2SH>B6SH & $\mathrm{P}<0.001$ \\
\hline \multirow[t]{2}{*}{ Exploratory behaviour } & \multirow[t]{2}{*}{$18.43 \pm 2.16$} & \multirow[t]{2}{*}{$3.33 \pm 2.19$} & \multirow[t]{2}{*}{$20.69 \pm 3.15$} & \multirow[t]{2}{*}{$3.87 \pm 3.09$} & $\mathrm{~B} 6 \mathrm{GH}>\mathrm{D} 2 \mathrm{GH}$ & $\mathrm{P}<0.001$ \\
\hline & & & & & $\mathrm{B} 6 \mathrm{SH}>\mathrm{D} 2 \mathrm{SH}$ & $\mathrm{P}<0.001$ \\
\hline \multirow[t]{2}{*}{ Locomotion } & \multirow[t]{2}{*}{$3.78 \pm 0.36$} & \multirow[t]{2}{*}{$1.97 \pm 0.45$} & \multirow[t]{2}{*}{$3.88 \pm 0.41$} & \multirow[t]{2}{*}{$1.07 \pm 0.35$} & $\mathrm{~B} 6 \mathrm{GH}>\mathrm{D} 2 \mathrm{GH}$ & $\mathrm{P}<0.001$ \\
\hline & & & & & $\mathrm{B} 6 \mathrm{SH}>\mathrm{D} 2 \mathrm{SH}$ & $\mathrm{P}<0.001$ \\
\hline
\end{tabular}

The plate was incubated at $80^{\circ} \mathrm{C}$ for 45 min to dryness in a thermostat in order to capture the glycans in the sample mixtures onto the beads via stable hydrazone bonds, and this was followed by successive washing using $200 \mu \mathrm{L}$ each of $2 \mathrm{M}$ guanidine- $\mathrm{HCl}$ in $16.6 \mathrm{mM} \mathrm{ABC}$ and water and $1 \%$ triethylamine in $\mathrm{MeOH}$ twice each. The acetyl capping of unreactive hydrazide functional groups was performed using $10 \%$ acetic anhydride in $\mathrm{MeOH}$ at $25^{\circ} \mathrm{C}$ for $30 \mathrm{~min}$. The remaining acetic anhydride was removed by a vacuum. In bead methyl esterification, each well was washed twice with $10 \mathrm{mM} \mathrm{HCl}, \mathrm{MeOH}$, and dioxane, consecutively, and $150 \mathrm{mM}$ MTT was then added, and the plate was incubated at $60^{\circ} \mathrm{C}$ for $90 \mathrm{~min}$. The beads were serially washed using $200 \mu \mathrm{L}$ of dioxane, water, methanol, and water. In order to obtain a perfect transamination reaction, 20 $\mu \mathrm{L}$ of $50 \mathrm{mM}$ BOA was added, followed by a treatment with $180 \mu \mathrm{L}$ of $2 \% \mathrm{AcOH}$ in $\mathrm{CH}_{3} \mathrm{CN}$ at $80^{\circ} \mathrm{C}$ for $45 \mathrm{~min}$, and an incubation at $80^{\circ} \mathrm{C}$ for $45 \mathrm{~min}$. Labeled $N$-glycans were finally eluted with $100 \mu \mathrm{L}$ water, and then directed to SweetBlot ${ }^{\mathrm{TM}}$, an automated glycan processing machine.

Mass Spectrometry (MALDI-TOF/MS): The recovered $N$-glycans labeled with BOA were finally eluted with100 $\mu \mathrm{L}$ water, analyzed after being spotted on MTP 384 target plates, and then crystallized by drying under a vacuum with an equivalent volume of the liquid matrix solution, $100 \mathrm{mM} \alpha$-cyano-4-hydroxycinnamic acid diethyl ammonium salt (CHCA-DEA) dissolved in buffer solution $\left(\mathrm{MeOH}: \mathrm{H}_{2} \mathrm{O}\right.$ : DMSO: $10 \mathrm{mM} \mathrm{NaOH}=50: 39$ : 10: 1). MALDI-TOF/MS data were then displayed on 

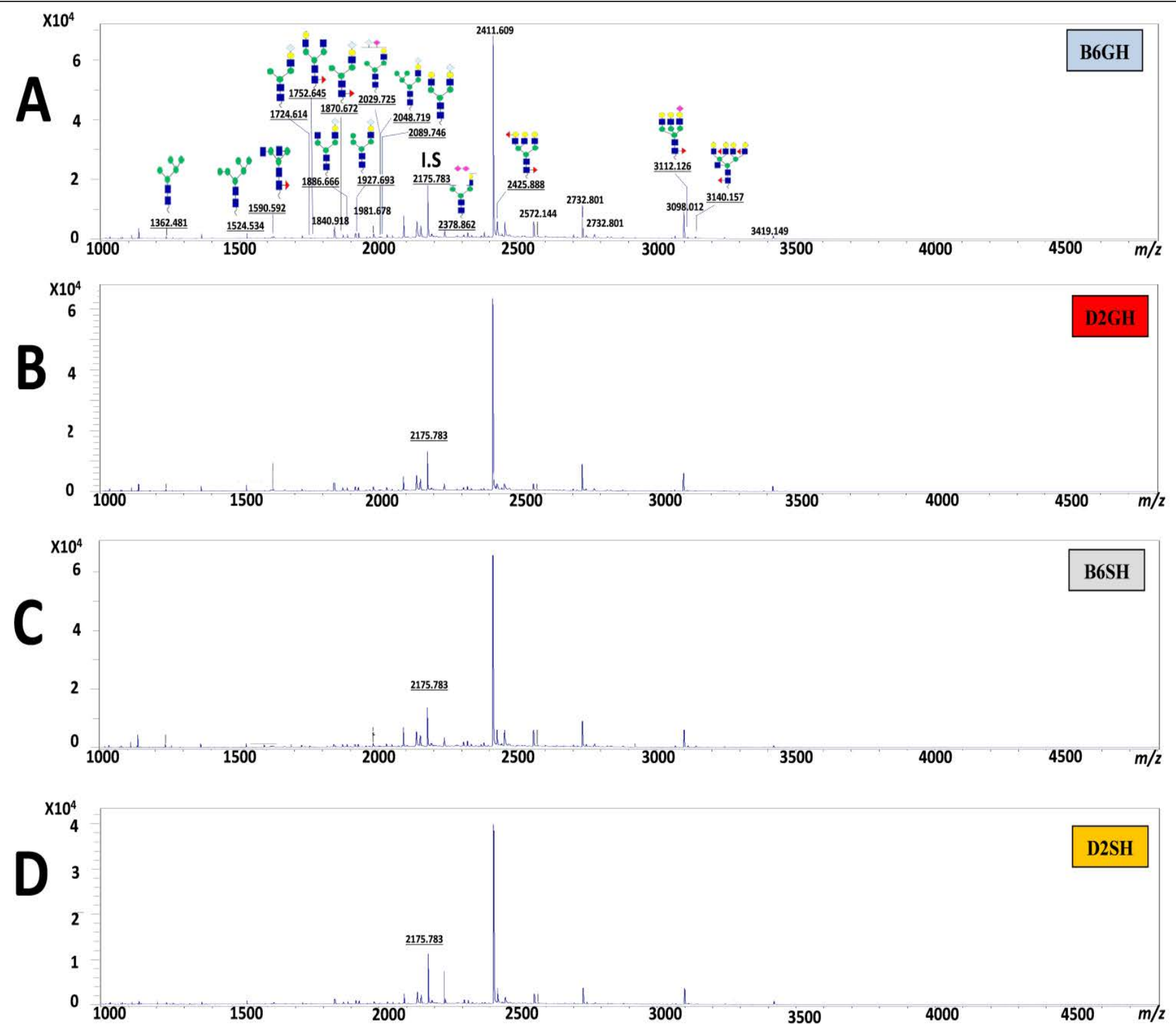

Figure S1: Representative MALDI-TOF/MS spectra showing typical $N$-glycan profiles of mouse serum. (A) in GH-C57BL/6, (B) in GH- DBA/2, (C) in SH- C57BL/6, and (D) in SH- DBA/2.I.S, internal standard at peak\# 2175. B6GH: GH-C57BL/6J; D2GH: GH-DBA/2; B6SH: SH-C57BL/6J; D2SH: SH-DBA/2.

an Ultraflex III, equipped with a reflector and controlled by the FlexControl 3.0 software package according to the general protocol. All spectral conditions were obtained using the reflector mode, ions generated by Smartbeam (pulsed UV solid laser, $\lambda_{\text {ex }}=355 \mathrm{~nm}, 50 \mathrm{~Hz}$ ) with $25 \mathrm{kV}$ as the acceleration voltage, $26.3 \mathrm{kV}$ as the reflector voltage, $160 \mathrm{~ns}$ as pulsed ion extraction in the positive mode, and typically totaling 1,000 shots of each spot.

Data Analysis: MS data were analyzed with the FlexAnalysis 3.0 software (BrukerDaltonik $\mathrm{GmbH}$, Bremen, Germany, $\mathrm{S} / \mathrm{N}=6$, and quality factor threshold of 30 ). 